University of the Pacific

Scholarly Commons

$9-11-2015$

\title{
4-Aminoquinoline-hybridization en route towards the development of rationally designed antimalarial agents
}

\author{
Raghu Raj \\ Guru Nanak Dev University \\ Kirkwood M. Land \\ University of the Pacific, kland@pacific.edu \\ Vipan Kumar \\ Guru Nanak Dev University, vipan_org@yahoo.com
}

Follow this and additional works at: https://scholarlycommons.pacific.edu/cop-facarticles

Part of the Biology Commons

\section{Recommended Citation}

Raj, R., Land, K. M., \& Kumar, V. (2015). 4-Aminoquinoline-hybridization en route towards the development of rationally designed antimalarial agents. RSC Advances, 5, 82676-82698. DOI: 10.1039/C5RA16361G https://scholarlycommons.pacific.edu/cop-facarticles/787

This Article is brought to you for free and open access by the All Faculty Scholarship at Scholarly Commons. It has been accepted for inclusion in College of the Pacific Faculty Articles by an authorized administrator of Scholarly Commons. For more information, please contact mgibney@pacific.edu. 


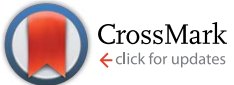

Cite this: RSC Adv., 2015, 5, 82676

Received 13th August 2015 Accepted 9th September 2015

DOI: $10.1039 / \mathrm{c} 5 \mathrm{ra} 16361 \mathrm{~g}$

www.rsc.org/advances

\section{4-Aminoquinoline-hybridization en route towards the development of rationally designed antimalarial agents}

\begin{abstract}
Raghu Raj, ${ }^{a}$ Kirkwood M. Land ${ }^{b}$ and Vipan Kumar*a
The resistance of Plasmodium falciparum, the causative agent of malaria, against quinine and chloroquine along with the lack of malaria vaccines has encouraged the development of various synthetic strategies towards biologically active scaffolds. An emerging strategy in medicinal chemistry, termed molecular hybridization, involves the covalent fusion of two or more drugs, active compounds, and/or pharmacophoric units into a hybrid compound, with fascinating activities and multiple but not essentially simultaneous pharmacological targets. 4-Aminoquinolines are considered as promising antimalarials and 4-aminoquinoline hybridization is considered as an attractive and feasible approach for the development of new molecular frameworks for averting and delaying the emergence of drug resistance along with improved efficacy. The present review article describes the recent developments on the 4-aminoquinoline-hybridization towards the development of new antimalarials.
\end{abstract}

${ }^{a}$ Department of Chemistry, Guru Nanak Dev University, Amritsar-143005, India. E-mail: vipan_org@yahoo.com; Fax: +91 1832258819 20; Tel: +91 183225880209 ext. 3320

${ }^{b}$ Department of Biological Sciences, University of the Pacific, Stockton, CA 95211, USA

\section{Introduction}

In the 20th century, the drug design approach accomplished the "one-target-one-drug" concept for the discovery of new drugs which, without any iota of doubt, will be dominant for many years. According to this concept, a single drug is designed for a

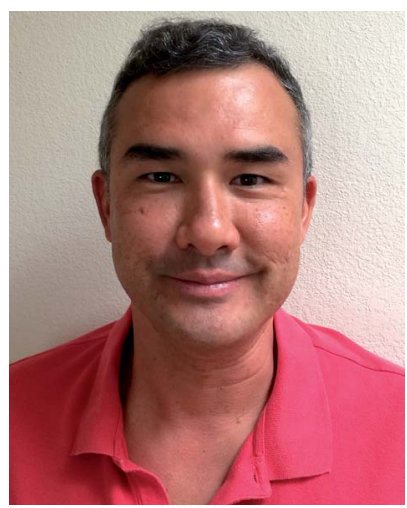

Dr Kirkwood Land is an Associate Professor in the Department of Biological Sciences at the University of the Pacific. Dr Land's research group explore interdisciplinary approaches to drug discovery. In particular, he is interested in strategies for the control of microorganisms, especially, bacteria and parasites associated with domesticated animals and humans. His work utilizes the One Health approach, which integrates an understanding of humans, animals, and the environment, to address current problems of infectious diseases. 
single target. Although numerous drugs have been designed and used clinically with good success using this approach, the strategy of one drug hammering one target remains inadequate for the treatment of several diseases, such as neurodegenerative syndromes, cardiovascular diseases, diabetes and cancer, which involve multiple pathogenic factors. ${ }^{1}$ Further, an ideal drug for one target does not always have clinical efficacy due to nonrecognition of the in vivo target or the inability to access the site of action. This target-based strategy does not always guarantee success since some selective drugs can work only in a selected number of patients. For example, Astra Zeneca's Iressa (gefitinib) is designed to treat lung cancer via targeting EGFR (Epidermal Growth Factor Receptor) protein. The drug provided an extremely potent response, but only in about $10 \%$ of the infected individuals. ${ }^{2,3}$ The ineffectiveness of single medicine paradigm necessitated the discovery of new paradigms where the drug therapy can block more than one target.

Among the different strategies developed to address the above issues, combination therapy was considered as a popular alternative in which a cocktail of drugs is co-administered in the form of two or more individual tablets to treat unresponsive patients. ${ }^{4}$ However, the advantage of combining therapeutic mechanisms of different drugs through this approach is compromised by patient compliance., ${ }^{5,6}$ The multi-component drug approach, involving co-formulation of two or more drugs in a single tablet, makes dosing regimens simpler, improves patient compliance, ${ }^{7,8}$ and even obviates the risk of drug-drug interactions present in combination therapy. This strategy has enhanced research and development ( $\&$ \& D), as evident by the launch of several multi-component drugs, including Caduet ${ }^{9}$ (atorvastatin + amlodipine) and Vytorin ${ }^{\mathbf{1 0}}$ (simvastatin + ezetimibe) for the treatment of cardiovascular disease, Coartem (artemether + lumefantrine) and Artekin (dihydroartemisinin +

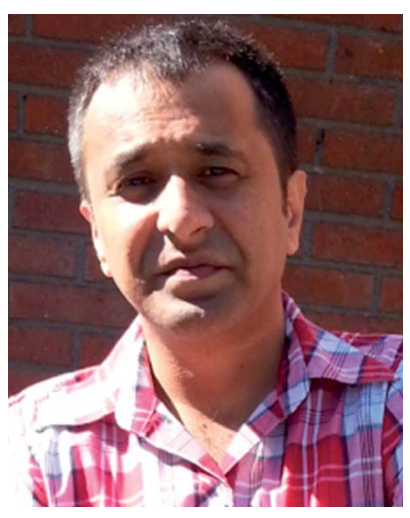

Dr Vipan Kumar Ph.D. has been working as an Assistant Professor in the Department of Chemistry, Guru Nanak Dev University, Amritsar, since 2009. $\mathrm{He}$ obtained his Ph.D. with Prof. Mohinder P. Mahajan in the Department of Applied Chemistry, Guru Nanak Dev University. In 2007, he moved to the University of Cape Town, South Africa, to pursue his postdoctoral studies with Prof. Kelly Chibale and extensively worked on molecular hybridization protocols for the preparation of molecular conjugates intended for HIV-malaria co-infections. At present, he is working on a research fellowship at the University of Umeå, Sweden. His research interests include the development of diverse synthetic protocols for the synthesis of novel molecular frameworks targeting tropical infections. He has also been engaged in the utilization of $\beta$-lactam synthon protocols for the synthesis of functionally decorated and biologically relevant heterocycles with medicinal potential. piperaquine) for the treatment of malaria, ${ }^{\mathbf{1 1}}$ and Atripla ${ }^{\mathbf{1 2}}$ (efavirenz + tenofovir/emtricitabine) against AIDS. However, highly complex pharmacokinetic (PK)/pharmacodynamic (PD) relationships in multi-component drugs due to the differences in relative rates of metabolism of drugs in different patients led to unpredictable variability. With the failure of these strategies, the development of new drug molecules that aim to modulate multiple targets simultaneously (polypharmacology) along with enhanced efficacy, improved safety, synthetic selectivity and economic accessibility represents a big challenge for the pharmaceutical sector.

Nowadays, molecular hybridization has successfully emerged as a promising tool for medicinal chemists and the drug design process, in which two or more different pharmacophoric units are covalently linked into a single hybrid molecule with superior affinity and efficacy as compared to the parent drugs. ${ }^{13-16}$ Molecular hybridization is beneficial as different targets are activated by a single molecule and is particularly interesting where treatment is limited to a few commercial drugs or in cases where the discovered bioactive compounds present high toxicity or pharmacokinetic and pharmacodynamic limitations. ${ }^{17,18}$ Hybrid molecules can be designed in different ways as follows:

- Metabolically stable hybrids, in which the desired molecules are tethered via linkers or spacers, which may or may not be stable under in vivo conditions. These conjugates interact preferably at more than one target with effects mostly additive or synergistic for a particular disease.

- Cleavable molecular hybrids, which get metabolized in vivo with the release of parent drug molecules and have different targets and mechanisms of action.

- Molecular hybrids can also be prepared via either fusing the drug molecules without introducing a linker or by merging the individual molecules by taking advantage of commonalities in their structure.

Today, much of the world's population is affected by infectious diseases that remain instrumental in debilitating poverty. According to the latest statistics published in 2012, 8.7 million people died worldwide in 2008 due to infectious diseases. ${ }^{19}$ People who lack food, shelter, security and social protection are more vulnerable to infectious diseases since they often lack the most basic measures of prevention and care. Pathogenic microorganisms, including bacteria, viruses, parasites or fungi, are the major cause of infectious disease and can spread directly or indirectly, from person to person. Among infectious diseases, malaria, tuberculosis (TB) and HIV/AIDS are high on the global agenda while other less important infections include Chagas disease, human African trypanosomiasis, trichomoniasis and leishmaniasis. ${ }^{20}$

Malaria is considered to be one of the most dangerous parasitic diseases because of its high morbidity and mortality, as well as its socio-economic impacts on the malaria-endemic region. It remains a chief cause of illness and death in tropical and subtropical countries, including Africa, Asia and South America. Approximately 90 percent cases of malaria are found in sub-Saharan Africa. According to the World Health Organization (WHO) report 2012, 3.4 billion people in 103 countries 
are at risk of infection with 207 million malaria cases and 627000 deaths, with the majority of victims being pregnant women or children less than the age of five years. ${ }^{21}$ The disease is caused by genus Plasmodium and, among 200 Plasmodium species, only five infect humans, viz. Plasmodium falciparum, $P$. vivax, $P$. ovale, $P$. malariae and $P$. knowlesi, with $P$. falciparum being the most virulent. Historically, the safe and cheap drug chloroquine (CQ) was extensively used for the treatment of malaria due to its excellent clinical efficacy, restricted host toxicity and simple cost-effective synthesis. CQ is believed to target ferriprotoporphyrin IX (FPIX) heme inside the digestive vacuole (DV) of the parasite. FPIX, being toxic to the parasite in its free state, is sequestered as a non-toxic crystalline hemozoin, also called "malarial pigment." CQ forms a complex with FPIX, resulting in the accumulation of toxic hematin (Fe(III)PPIX) within the digestive vacuole and instigating the death of the parasite. ${ }^{22-29}$ However, the onset of resistant strains to the available antimalarial drugs, including $\mathbf{C Q}$, is responsible for the global rise of malaria and offers a strong impetus for the development of new antimalarial drugs. ${ }^{30,31}$ The development of resistance of $P$. falciparum to $\mathbf{C Q}$ is mainly attributed to the mutations in the P. falciparum chloroquine resistant transporter gene (PfCRT), a protein involved in the efflux of drug and proton equilibrium across the membrane of the digestive vacuole, resulting in poor accumulation of $\mathbf{C Q}$ in the acidic food vacuole of the parasite. ${ }^{32,33}$ Currently, the WHO recommends artemisinin based combination therapy (ACT) comprising artemisinin and its semi-synthetic derivatives in combination with existing drugs for the treatment of malaria. This drug regimen is considered successful against both $\mathbf{C Q}$-sensitive as well as CQ-resistant strains of malaria. ${ }^{34-36}$ However, recent reports of the development of clinical resistance to ACT in Southeast Asia threaten this combination therapy. ${ }^{37}$

4-Aminoquinoline hybridization is now considered an attractive and viable strategy for preventing and delaying the emergence of drug resistance along with the improvement in efficacy. ${ }^{38-42}$ The success of the quinoline-hybridization strategy was exemplified by several potential antimalarials, such as trioxaferroquines, ${ }^{43}$ trioxaquines, ${ }^{44}$ artemisinin-quinine hybrid, ${ }^{45}$ 4-aminoquinoline based tetraoxanes, ${ }^{46}$ clotrimazole-based4 -aminoquinoline $\mathrm{e}^{47}$ and isatin-4-aminoquinoline hybrids. ${ }^{48}$ The present review article encompasses the recent developments on the utility of 4-aminoquinoline-hybridization towards the development of novel antimalarials. A special focus has been given to the structure-activity relationship, $\mathrm{IC}_{50}$ values against CQ-sensitive and resistant strains, in vivo evaluation data and cytotoxic studies of the promising candidates that have emerged from this strategy.

\section{4-Aminoquinoline-based antimalarial conjugates}

\subsection{4-Aminoquinoline-chalcone conjugates}

Chalcones and dienones are structurally linked compounds that have been revealed to exhibit notable in vitro and in vivo antimalarial activity ${ }^{49-52}$ by acting as inhibitors of either

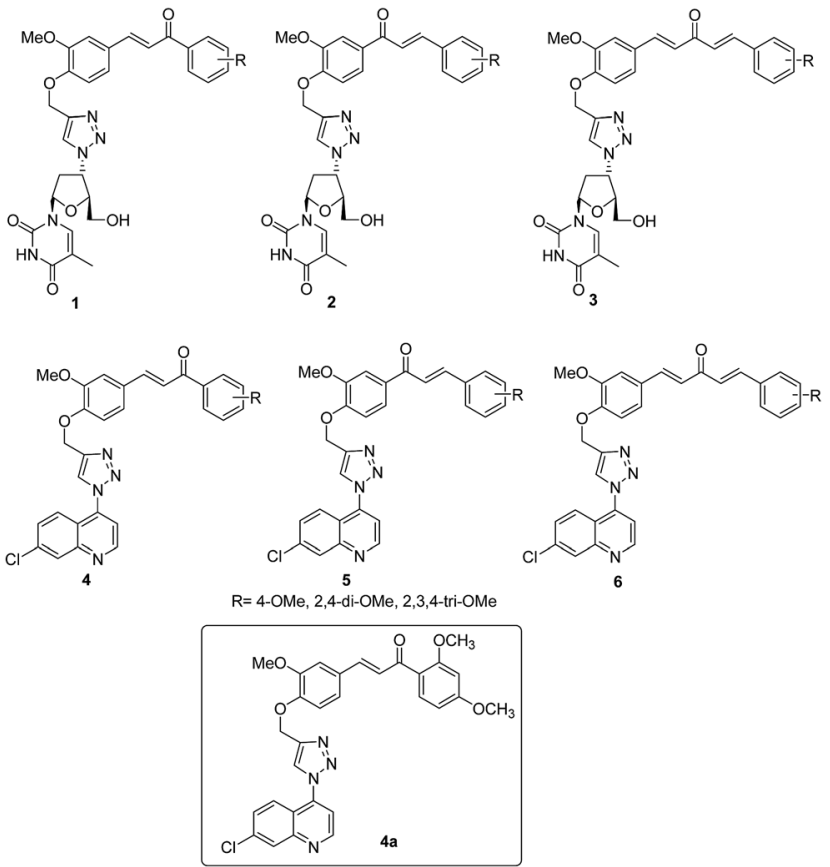

Fig. 1 General structures for triazole-linked chalcone and dienone hybrid compounds comprising AZT and aminoquinoline with the most active hybrid being 4 a.

plasmodial aspartate proteases, ${ }^{53}$ cysteine proteases $^{54}$ or permeability pathways initiated into erythrocyte cell membranes by the malaria parasite. ${ }^{55}$ Chibale and co-workers applied the molecular hybridization strategy via $\mathrm{Cu}(\mathrm{I})$-catalyzed cycloaddition reaction of terminal alkynes and azides for the synthesis of series of $1 H-1,2,3$-triazole linked chalcone and dienone conjugates containing aminoquinoline and nucleoside templates (Fig. 1)..$^{56}$ The synthesized hybrids were screened for their antimalarial activity against the CQ-sensitive (D10) and CQ-resistant (Dd2 and W2) strains of P. falciparum. Notably, the azidothymidine (AZT) conjugates with both chalcones and dienones did not show any improvement in the antimalarial activity over their acetylenic precursors while retention of activity was observed in most cases. The quinolinehybridization approach led to the identification of highly

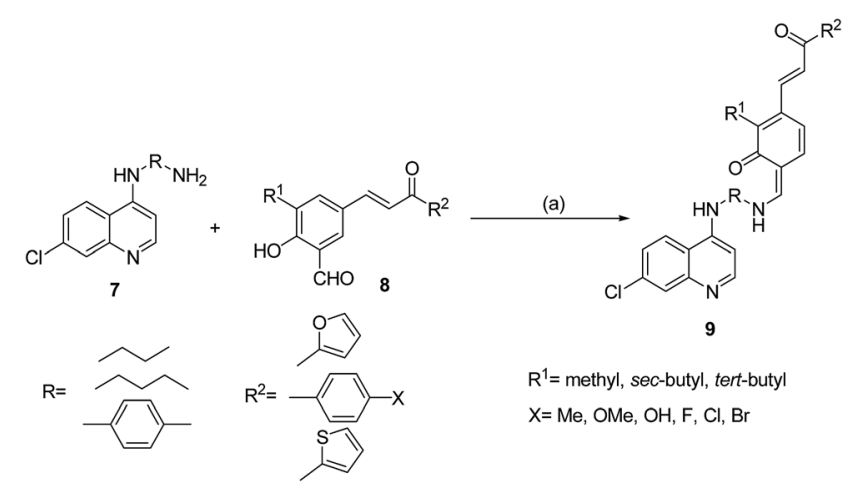

Scheme 1 Reagents and conditions: (a) ethanol, rt, $10 \mathrm{~min}$. 


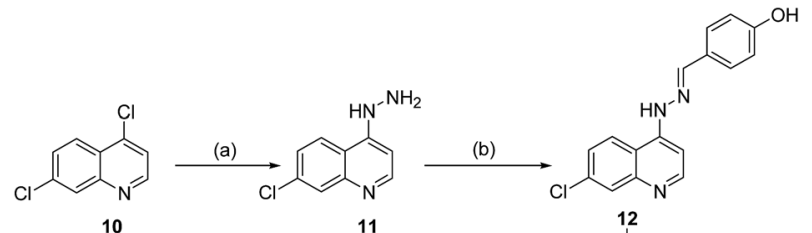

11
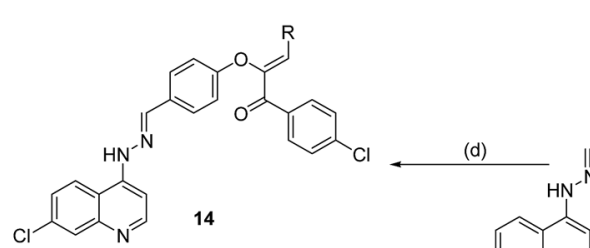

14

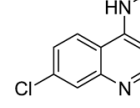

(c)
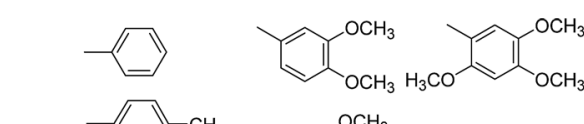

$\mathrm{R}=$
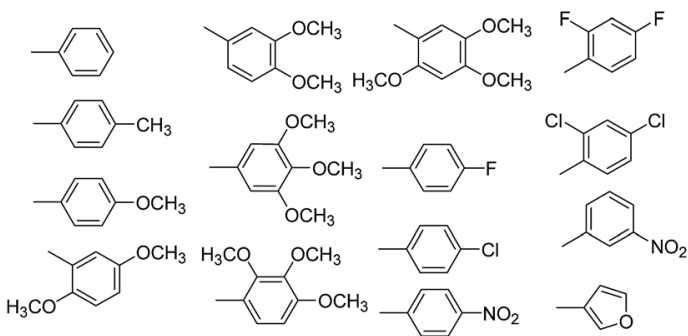

Scheme 2 Reagents and conditions: (a) hydrazine hydrate, EtOH, reflux, 8 h; (b) 4-hydroxybenzaldehyde, EtOH, reflux, 2-3 h; (c) 4-chlorophenacyl bromide, $\mathrm{K}_{2} \mathrm{CO}_{3}$, acetonitrile, rt, 2 h; (d) different substituted aldehydes, $10 \%$ methanolic $\mathrm{KOH}$, rt.

potent hybrids, with the most active conjugate 4 a having submicromolar $\mathrm{IC}_{50}$ values of $0.04,0.07$ and $0.09 \mu \mathrm{M}$ against tested D10, Dd2 and W2 strains of $P$. falciparum, respectively. Cytotoxicity against the Chinese Hamster Ovarian cell line was determined and the hybrid 4 a proved to be non-cytotoxic even at the highest concentration tested $(100 \mu \mathrm{M})$.

Sashidhara and co-workers utilized an uncommon approach towards the synthesis of keto-enamine tethered chalcone4-aminoquinoline conjugates (9). ${ }^{57}$ The synthesized conjugates were evaluated for their antimalarial potential against a CQ-sensitive (3D7) strain of $P$. falciparum (Scheme 1). The promising compounds from in vitro assay were further screened for their antimalarial efficacy against $P$. yoelii (CQ-resistant N-67 strain) in Swiss mice. Two of the synthesized conjugates displayed suppression of 99.9 percent parasitemia on day 4 . Mechanistically, the test compounds exhibited an antimalarial mode of action similar to that of $\mathbf{C Q}$, as confirmed by inhibition of $\beta$-hematin formation studies.

The above work was further extended towards the synthesis of 4-aminoquinoline-chalcone conjugates by following the sequence of synthetic steps shown in Scheme 2 along with their antimalarial evaluation. ${ }^{58}$ Most of the synthesized conjugates showed improved antimalarial profile against the Q-resistant K1 strain when compared to CQ. Structure activity relationship (SAR) studies demonstrated the dependence of the activity profile on the substitution pattern as well as the number of substituents present on the phenyl ring. The presence of electron releasing groups $\left(\mathrm{R}=-\mathrm{CH}_{3},-\mathrm{OCH}_{3}\right)$ has been shown to improve the activity profiles while replacing them with electron withdrawing groups $\left(\mathrm{R}=-\mathrm{Cl},-\mathrm{NO}_{2}\right)$ reduced the antimalarial activity, with the exception of fluorine. $\beta$-Hematin studies were also carried out for the synthesized conjugates with the three compounds being most active in inhibition of $\beta$-hematin formation with $\mathrm{IC}_{50}$ values of $3.46,3.52$ and $3.74 \mu \mathrm{g} \mathrm{ml}^{-1}$.

Insuasty et al. utilized 7-chloroquinoline-amino-chalcone hybrids (16) for the preparation of a series of $N$-acetyl and $\mathrm{N}$-formyl-pyrazoline derivatives (17 and 18) in acceptable to good yields via a cyclo-condensation reaction using hydrazine hydrate under acidic conditions (Scheme 3). The synthesized conjugates were bio-evaluated for their antimalarial and anticancer profiles. ${ }^{59}$ The anticancer profiles against 60 cell lines revealed that $\mathrm{GI}_{50}$ values for most of these conjugates range from 0.13 to $0.99 \mu \mathrm{M}$. The antimalarial activity performed against the NF54 strain of $P$. falciparum revealed that one of the $N$-formyl-pyrazoline derivatives, 18a, exhibited $50.8 \%$ inhibition.

A series of 4-aminoquinolinyl-chalcone amides (21) were synthesized by N'Da through condensation of carboxylic acidfunctionalized chalcone with aminoquinolines (Scheme 4) along with their screening against CQ-sensitive (3D7) and CQ-resistant (W2) strains of $P$. falciparum. Cytotoxicity against the WI-38 cell line of normal human fetal lung fibroblast was also evaluated. ${ }^{60}$ The $\mathrm{IC}_{50}$ values of the synthesized conjugates ranged between 0.04-0.5 $\mu \mathrm{M}$ and 0.07-1.8 $\mu \mathrm{M}$ against 3D7 and $\mathrm{W} 2$, respectively. SAR studies revealed the increased antimalarial activity of the amides with the increase in lipophilicity and alkyl chain length. Moderate to high toxicity towards mammalian cells was observed for these conjugates. The most active compound featuring 1,6-diaminohexane as the linker was twofold more potent than CQ against the 3D7 and W2 strains despite its predicted high lipophilicity, low solubility and poor absorption properties.

\subsection{4-Aminoquinoline-pyrimidine conjugates}

Rawat and co-workers reported the preparation of a library of 4-aminoquinoline-pyrimidine conjugates in an attempt to

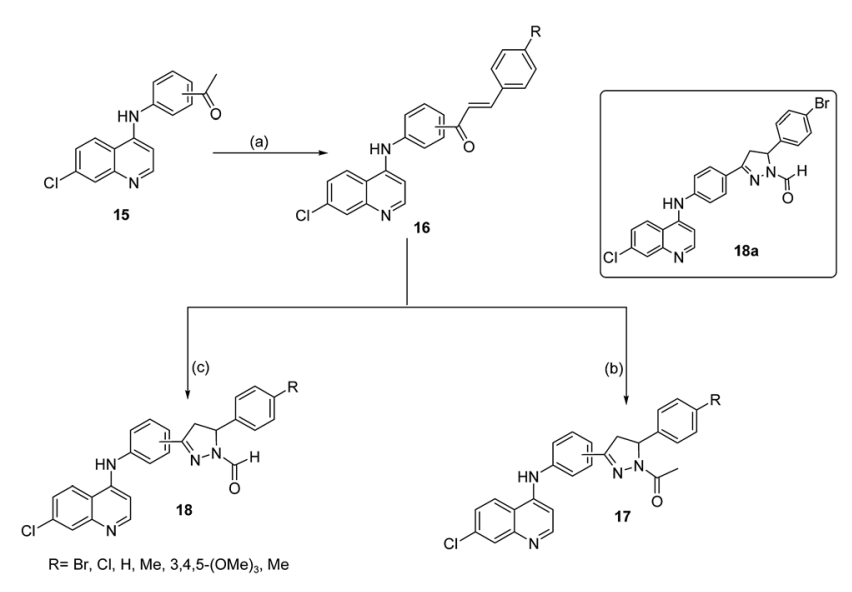

Scheme 3 Reagents and conditions: (a) $\mathrm{KOH}, \mathrm{MeOH}, \mathrm{rt}$; (b) hydrazine hydrate, acetic acid, reflux; (c) hydrazine hydrate, formic acid, DMF, reflux. 


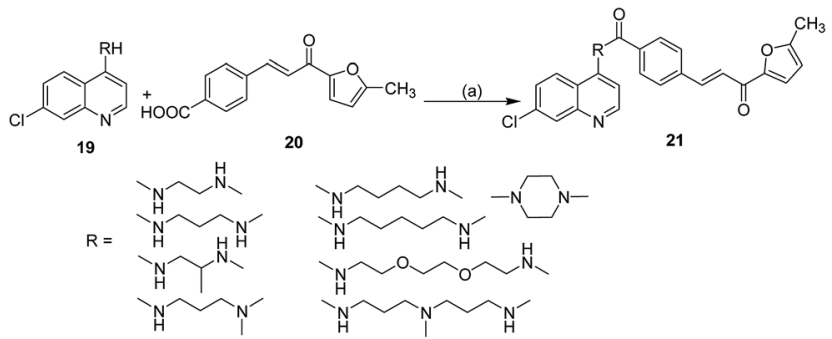

Scheme 4 Reagents and conditions: (a) CDI, DCM : DMF, rt, $24 \mathrm{~h}$.

search for more active molecules with effectiveness against both CQ-sensitive and CQ-resistant strains of P. falciparum. ${ }^{61}$ Among the synthesized compounds, eleven conjugates displayed enhanced antimalarial activity compared to CQ against both D6 and W2 strains, while four conjugates $(\mathbf{2 2}, \mathbf{2 3}, \mathbf{2 4}$, and 25, Fig. 2) showed better activity against both CQ-sensitive as well as CQ-resistant (D6 and W2) strains of P. falciparum in comparison to pyrimethamine. Most of the conjugates were found to be noncytotoxic up to a concentration of $60 \mu \mathrm{M}$, while others showed mild toxicities. Most of the conjugates exhibited a high selective index; compounds 22 and 25, with $\mathrm{IC}_{50}$ values of 0.005 and $0.006 \mu \mathrm{M}$, respectively, against the D6 strain and 0.03 and 0.06 $\mu \mathrm{M}$, respectively, against the $\mathrm{W} 2$ strain of $P$. falciparum, were selected for in vivo studies, and showed outstanding activity in a mouse model of $P$. berghei without any toxicity.

In a recent communication, Singh and co-workers showed the synthesis of molecular conjugates 27 based on 7-chloro-4aminoquinoline (7) and 2-aminopyrimidine (26) motifs, as shown in Scheme $5 .^{62}$ The rationale behind using 2 -aminopyrimidine was because of its well established antimalarial potential, as evidenced by the structurally related drug pyrimethamine. The antiplasmodial profiles of the synthesized conjugates were in the nanomolar range, with the most potent hybrid exhibiting an $\mathrm{IC}_{50}$ value of $3.6 \mathrm{nM}, 56$-fold less compared to $\mathbf{C Q}$ against the $\mathbf{C Q}$-resistant K1 strain. Almost all of the synthesized compounds were cytotoxic and the binding studies with DNA implied their strong affinity for target parasite type AT rich pUC18 DNA. The active conjugates also showed good inhibitory activity for $\beta$-haematin formation, suggestive of the fact that the observed antiplasmodial potential of the conjugates in the present case is because of their ability to act on multiple targets.
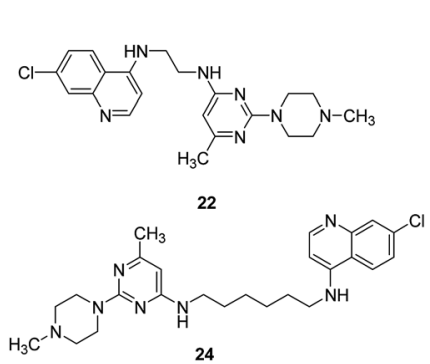

24

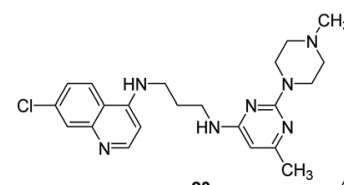

23

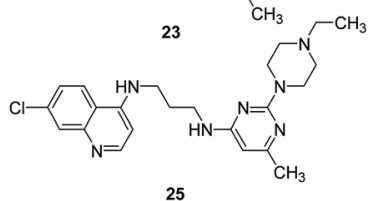

25

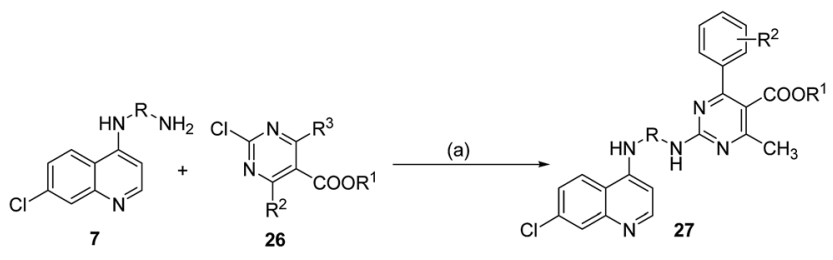

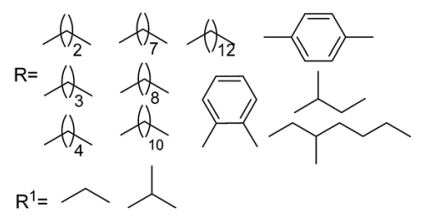

$\mathrm{R}^{2}=\mathrm{H}, 2-\mathrm{NO}_{2}, 3-\mathrm{NO}_{2}, 4-\mathrm{NO}_{2}$,

Scheme 5 Reagents and conditions: (a) $\mathrm{K}_{2} \mathrm{CO}_{3}, \mathrm{THF}, 48 \mathrm{~h}$, rt.

A range of 7-chloroquinoline-pyrimidine hybrids, viz. 30 and 31, were synthesized by N'Da and co-workers via aromatic nucleophilic substitution reaction of 4 -aminoquinolines with 2,6-diamino-4-chloropyrimidine and their antiplasmodial potential evaluated. The conjugates were evaluated alongside CQ, pyrimethamine (PM) and their fixed combinations viz. CQ/PM (1:1) and CQ/PM (1:4) against the CQ-susceptible D10 and -resistant Dd2 strain of P. falciparum (Scheme 6). ${ }^{63}$ The cytotoxic profiles of the synthesized scaffolds were also evaluated against the Chinese Hamster Ovarian (CHO) cell line. All the synthesized hybrids showed activity against both D10 and Dd2 strains with good selective index. The most potent compound of the series $\mathbf{3 0}$ with piperazine as the linker showed comparable activity to that of PM and CQ against the D10 strain $\left(\mathrm{IC}_{50}=0.07 \mu \mathrm{M}\right)$ while a three-fold better potency than that for CQ against Dd2 strain $\left(\mathrm{IC}_{50}=0.157 \mu \mathrm{M}\right)$ was observed.

Further, a library of piperazine tethered 4-aminoquinolinepyrimidine conjugates was prepared and screened for their antimalarial profiles over CQ-sensitive (D6) and CQ-resistant (W2) strains of P. falciparum while cytotoxicity was evaluated against the mammalian Vero cell line. ${ }^{64}$ Nine conjugates were shown to have superior antimalarial potency against both the

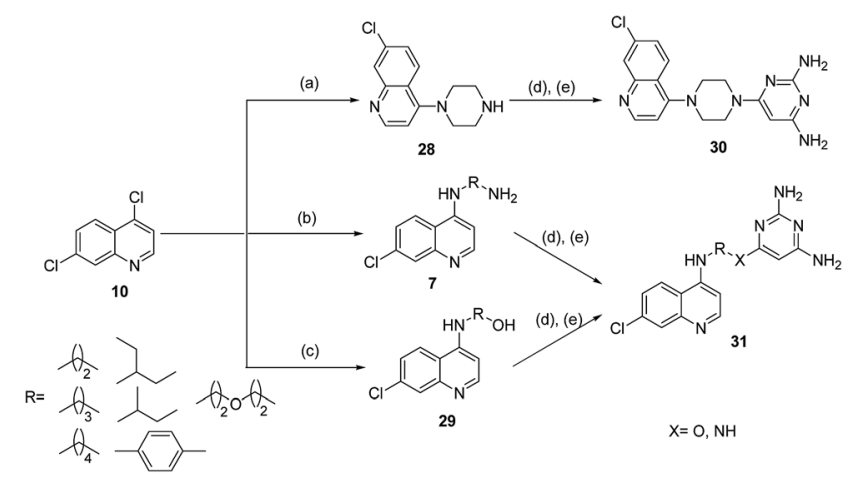

Scheme 6 Reagents and conditions: (a) piperazine, DMF, $80-135^{\circ} \mathrm{C}$, $5 \mathrm{~h}$; (b) diaminoalkane/aryldiamine, neat or DMF, $80-150^{\circ} \mathrm{C}, 24 \mathrm{~h}$; (c) aminoalcohol, neat, $120^{\circ} \mathrm{C}, 24 \mathrm{~h}$; (d) NaH, DMF, rt, 1 h; (e) 2,6-diamino-4-chloropyrimidine, DMF, $135^{\circ} \mathrm{C}, 16-24 \mathrm{~h}$.
Fig. 2 Most potent 4-aminoquinoline-pyrimidine conjugates 22, 23, 24 and 25. 


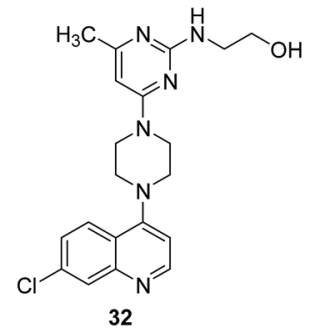

$\mathrm{IC}_{50}=0.13 \mu \mathrm{M}$ against $\mathrm{D} 6$ strain $\mathrm{IC}_{50}=0.14 \mu \mathrm{M}$ against $\mathrm{W} 2$ strain

Fig. 3 Most potent piperazine tethered 4-aminoquinoline-pyrimidine conjugate 32 and $C Q$.

strains with $\mathrm{IC}_{50}$ values ranging from 0.13 to $0.14 \mu \mathrm{M}$. Out of these, compound 32 (Fig. 3) proved to be the most potent among the synthesized conjugates and its antimalarial activity was found to be 2.5 fold more than that of the standard drug CQ. All the synthesized conjugates were non-cytotoxic against the Vero cell line.

The above work was further extended for the preparation of 4-aminoquinoline-pyrimidine hybrids 34, as shown in Scheme 7. All the synthesized compounds were screened for their antiplasmodial activity against D6 and W2 strains of $P$. falciparum, depicting activity in the nano-molar range. ${ }^{65}$ Numerous compounds were found to be nontoxic to the mammalian cell lines and showed promising in vitro antimalarial activity over both CQ-sensitive and -resistant strains with high values of selective index. The most potent conjugate $34 a$ with an $\mathrm{IC}_{50}$ value of 0.033 and $0.058 \mu \mathrm{M}$ against D6 and W2 strains of $P$. falciparum, respectively, was chosen for in vivo studies and exhibited significant suppression of parasitemia. The heme binding studies confirmed heme as one of the possible targets of these hybrids with heme forming a stable $1: 1$ complex with these conjugates. Potent compounds from in vitro antimalarial activity data were selected for molecular docking simulations and showed good interaction with the binding sites of PfDHFR.

\subsection{4-Aminoquinoline-triazine conjugates}

Rawat et al. described the synthesis of a library of 4-aminoquinoline-1,2,3-triazoles (36) and 4-aminoquinoline-

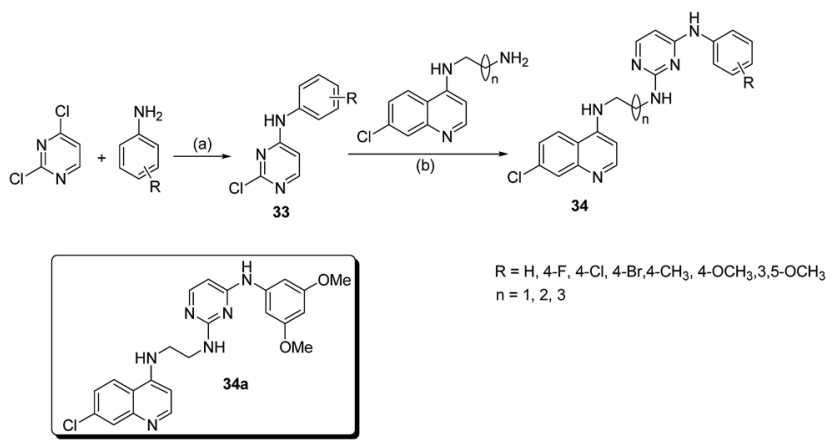

Scheme 7 Reagents and conditions: (a) TEA, EtOH, rt, overnight; (b) $\mathrm{K}_{2} \mathrm{CO}_{3}, \mathrm{NMP}, 140-150{ }^{\circ} \mathrm{C}, 10-12 \mathrm{~h}$.

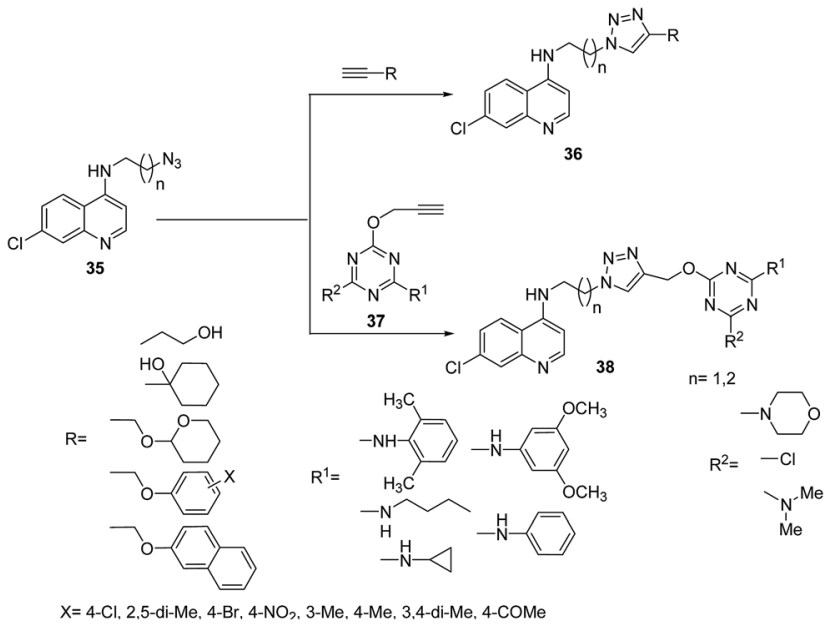

Scheme 8 Reagents and conditions: (a) $\mathrm{CuSO}_{4} \cdot 5 \mathrm{H}_{2} \mathrm{O}$, sodium ascorbate, $t-\mathrm{BuOH}: \mathrm{H}_{2} \mathrm{O}(1: 1), 40^{\circ} \mathrm{C}, 3 \mathrm{~h}$.

1,2,3-triazole-1,3,5-triazine conjugates (38) by utilizing Huisgen1,3-dipolar cycloaddition reaction of 4-azido-7-chloroquinoline with variedly substituted terminal alkynes (Scheme 8). ${ }^{66}$ It has been hypothesized that amalgamation of a basic nucleus viz. triazine with 4-aminoquinoline would enhance the accumulation of the conjugate in the digestive food vacuole, targeting $P$. falciparum dihydrofolate reductase (PfDHFR). However, the antimalarial profile of the synthesized conjugates against CQ-sensitive D6 and CQ-resistant W2 strains revealed that none of the hybrids exhibited better activity than that of CQ.

The above study was further extended by Chauhan and co-workers in the synthesis of 4-anilinoquinoline-triazine derivatives (40 and 41) using readily available and cheap starting materials (Scheme 9). The compounds were assessed for their antiplasmodial efficacy against the CQ-sensitive 3D7 strain of $P$. falciparum as well as for their cytotoxicity towards the mammalian Vero cell line. ${ }^{67}$ The most promising and noncytotoxic compounds from the preliminary studies were chosen for in vivo screening in Swiss mice infected with the

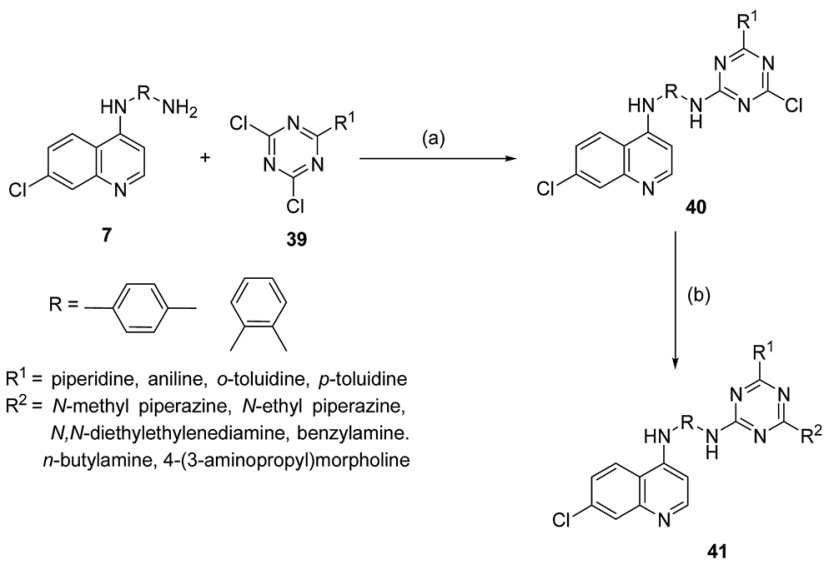

Scheme 9 Reagents and conditions: (a) mono-substituted triazines, THF, reflux, 8 h; (b) various amines, THF, reflux, 5 h. 


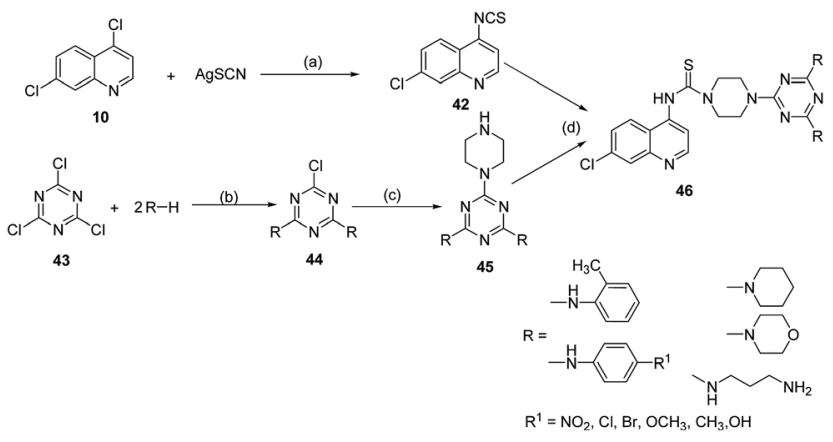

Scheme 10 Reagents and conditions: (a) reflux with stirring for $18 \mathrm{~h}$ at 90-120 ${ }^{\circ} \mathrm{C}$; (b) dry acetone, $0-5{ }^{\circ} \mathrm{C}, 1 \mathrm{~h}$, then $40-45^{\circ} \mathrm{C}$ for a further $3 \mathrm{~h}$; (c) piperazine, 1,4-dioxane, $120-130{ }^{\circ} \mathrm{C}, 5-6 \mathrm{~h}$; (d) dry acetone, $40-45^{\circ} \mathrm{C}, 18 \mathrm{~h}$

CQ-resistant N-64 strain of $P$. yoelli. These studies resulted in the identification of two orally active compounds with piperidine functionality. The results obtained are suggestive of the fact that the antimalarial activity in these conjugates can be attributed to the metabolic stability of piperidine functionality. The selected conjugates were further investigated for their ability to inhibit $\beta$-hematin formation. All the tested hybrids exhibited better inhibitory activities of $\beta$-haematin formation $\left(\mathrm{IC}_{50}\right.$ ranging from 2.65 to $\left.3.16 \mu \mathrm{M}\right)$ than $\mathrm{CQ}\left(\mathrm{IC}_{50}=3.65 \mu \mathrm{M}\right)$, which revealed that, apart from targeting heme, there might be the possibility of other mechanisms of action.

The use of triazine functionality for the synthesis of molecular conjugates with antimalarial potential was further elaborated by Bhat and co-workers in the synthesis of variedly functionalized 1,2,3-triazine-4-aminoquinoline conjugates (46) attached via thiourea functionality as the linker (Scheme 10). The determination of their antimalarial efficacy was done against the CQ-sensitive (3D7) and CQ-resistant (RKL-2) strains of P. falciparum. ${ }^{68}$ The observed SAR revealed a preference for electron withdrawing substituents, viz. bromo and hydroxyl groups, while the presence of electron donating substituents, including methyl and methoxy, adversely affected the activity profiles. The observed activities were further substantiated by

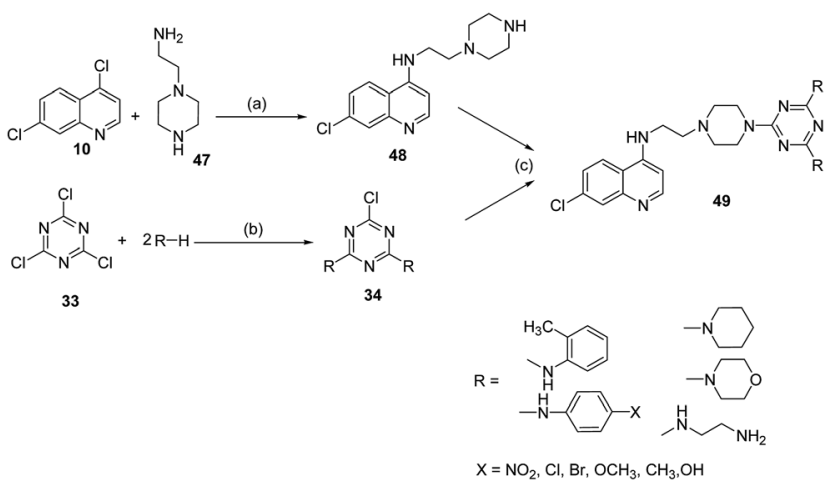

Scheme 11 Reagents and conditions: (a) reflux $1 \mathrm{~h}$ at $80^{\circ} \mathrm{C}$ followed by $6-8 \mathrm{~h}$ at $120-130{ }^{\circ} \mathrm{C}$; (b) 1,4 -dioxane $0-5^{\circ} \mathrm{C}, 1 \mathrm{~h}, 40-45^{\circ} \mathrm{C}, 3 \mathrm{~h}$; (c) 1,4-dioxane, $120-130{ }^{\circ} \mathrm{C}, 6-7 \mathrm{~h}$. docking simulations performed by using the ligand fit module within Discovery Studio 2.5 on wild and quadruple mutant strains of $P$. falciparum dihydrofolate reductase thymidylate synthase (pf-DHFR-TS).

Further extension of the above work resulted in the synthesis of 4-aminoquinoline-1,3,5-triazine derivatives (49) connected through non-ionizable covalent linkers (Scheme 11) ${ }^{69}$ The antiplasmodial profile again showed a similar SAR to that observed earlier with a marked preference for electron withdrawing substituents for good activity. Further, addition of basic moieties, such as morpholine, piperidine and 1,3-diaminopropane, in the synthesized conjugates was shown to enhance the antimalarial activity. The evaluation results were correlated using docking studies carried out on wild and quadruple mutants pf-DHFR-TS. In the wild type strain, most of the hybrids revealed a hydrophobic interaction between the phenyl ring of conjugates with the Phe58 residue as well as a $\sigma-\pi$ interaction between the 1,3,5-triazine ring and the Leu46 residue. Similarly, a hydrophobic interaction was observed between the phenyl ring and Phe58 in addition to H-bond formation between the 1,3,5triazine ring and Ser111 in the quadruple mutant of pf-DHFR-TS. Further, it was observed that the quinoline moiety was engaged in the formation of a $\pm \pi$ interaction with Arg59.

\subsection{4-Aminoquinoline-thiazolidine conjugates}

An alternative pathway for obtaining new and promising antimalarial compounds was disclosed by Kouznetsov and co-workers. A library of 21 heterocyclic scaffolds consisting of either $N$-(aminoalkyl)thiazolidin-4-one-4-aminoquinoline conjugates (50) or substituted $N$-benzylamino-7-chloroquinolines (51) was prepared by following the sequence of steps depicted in Scheme $12 .^{70}$ The antimalarial evaluation against 3D7 and Dd2 strains showed that four of the $N$-benzylamino-7chloroquinoline derivatives (51) are up to 3 -fold more active compared to the standard drug, CQ. Non-specific cytotoxicity assay on J774 murine macrophages and HepG2 cells (human hepatocellular carcinoma cell line) proved their high selectivity index and hence the potential for in vivo evaluation.

Chauhan and co-workers recently explored the synthesis of 4aminoquinoline-rhodanine hybrids along with their in vitro

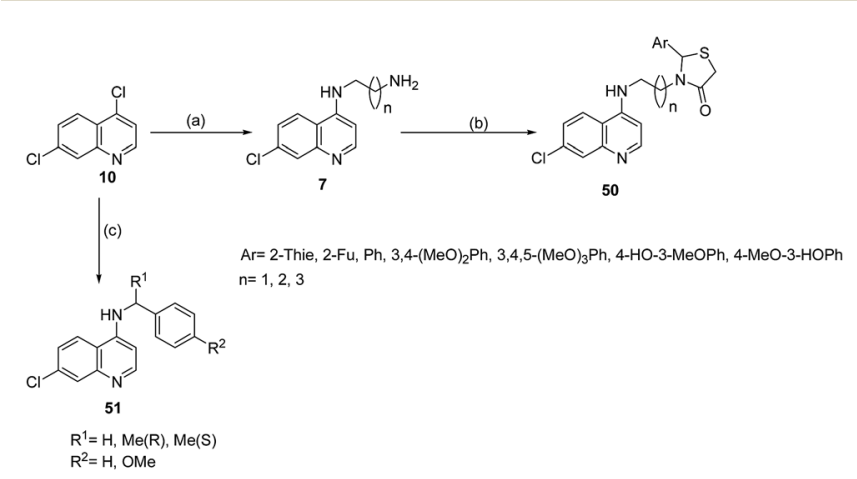

Scheme 12 Reagents and conditions: (a) diaminoalkane, $80^{\circ} \mathrm{C}$ for $1 \mathrm{~h}$, $140-150^{\circ} \mathrm{C}$ for 6-7 h; (b) ArCHO, $\mathrm{HSCH}_{2} \mathrm{COOH}, \mathrm{PhMe}, 4-5 \mathrm{~h}$ reflux; (c) substituted $\mathrm{N}$-benzylamine, $\mathrm{K}_{2} \mathrm{CO}_{3}, \mathrm{DMF}, 140{ }^{\circ} \mathrm{C}, 10 \mathrm{~h}$. 


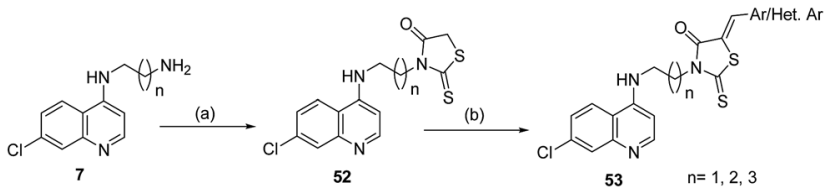

Scheme 13 Reagents and conditions: (a) $\mathrm{CS}_{2}, \mathrm{BrCH}_{2} \mathrm{COOC}_{2} \mathrm{H}_{5}$, acetonitrile, rt, 3-5 h, (b) acetic acid, ammonium acetate, aromatic/ heteroaromatic aldehydes, $90^{\circ} \mathrm{C}, 4-6 \mathrm{~h}$.

antimalarial efficacy against $\mathrm{K} 1$ and 3D7 strains of $P$. falciparum, as well as their cytotoxicity against the Vero cell line (Scheme 13). ${ }^{71}$ Although none of the hybrids was more potent than CQ against the 3D7 strain, four of the tested conjugates showed antimalarial activity comparable to $\mathbf{C Q}$ with $\mathrm{IC}_{50}$ values in the range of 13.2$45.5 \mathrm{nM}$ against $\mathrm{K} 1$ strain along with high selectivity. Furthermore, some of the hybrids were also tested for their anti-mycobacterial potential against the $\mathrm{H}_{37} \mathrm{Rv}$ strain of $M$. tuberculosis with three of the potent compounds exhibiting minimum inhibitory concentration (MIC) values of $6.25 \mu \mathrm{M}$. In addition, inhibition of $\beta$ hematin formation studies disclosed that the test conjugates were more active than CQ in the inhibition of hemozoin formation, clearly validating their mechanism of action.

A series of differently substituted 4-aminoquinolines having thiazolidin-4-ones (54), [1,3]thiazinan-4-ones (55) or 2,3-dihydrobenzo[e][1,3]thiazin-4-ones (56) at the terminal amino functionality was synthesized via a three-component reaction between 4-aminoquinolines, aryl aldehydes and appropriate mercapto acids (Scheme 14). ${ }^{72}$ Among the compounds tested against the NF-54 strain of $P$. falciparum, nine showed $\mathrm{IC}_{50}$ values ranging between $0.013-0.98 \mu \mathrm{M}$. The promising compounds from in vitro assay were further selected for in vivo activity testing in Swiss mice using N-67 strain of $P$. yoelli. The most potent compound from in vivo analysis suppressed parasitemia by 81 percent on day 4 compared to 100 percent suppression exhibited by CQ. SAR studies showed that the lateral side chain modification of 4-aminoquinoline in these conjugates was well tolerated with the best results obtained with a two- or three-carbon chain as the linker. The biochemical studies confirmed their association with hematin and hence confirmed the mechanism of action of the test compounds.

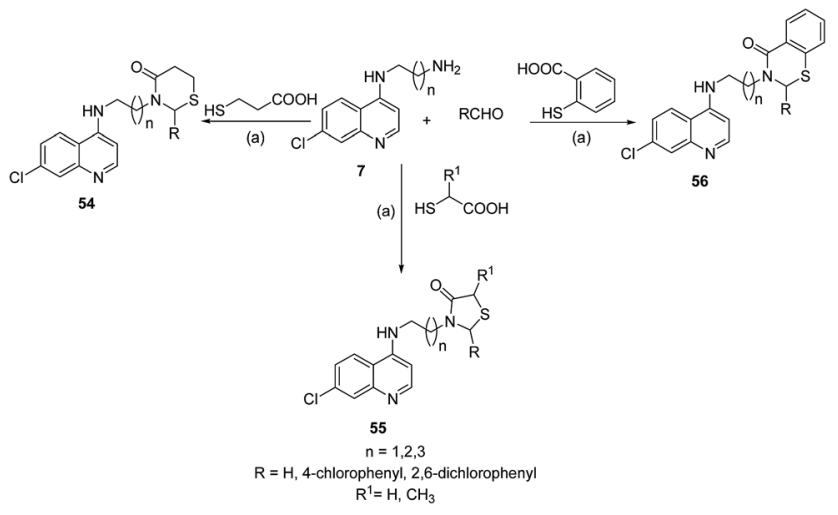

Scheme 14 Reagents and conditions: (a) DCC, THF, rt or PhMe, reflux.

\subsection{4-Aminoquinoline-clotrimazole conjugates}

With the aim of developing new antimalarials, in a recent communication Gemma and co-workers identified a new polyaromatic antimalarial pharmacophore based on a clotrimazole scaffold.$^{73}$ Clotrimazole is a popular antimycotic drug with weak antimalarial activity $\left(\mathrm{IC}_{50}=0.55 \mu \mathrm{M}\right)$ against the W2 strain of P. falciparum. However, its peculiar structural features, viz. (i) the presence of an imidazole nucleus, identified to mediate electron transfer reaction and (ii) the triphenylmethyl system known to stabilize radical intermediate, makes it an ideal candidate to interact with the haemoglobin-derived ferroprotoporphyrin (Fe(II)-FP) complex in the food vacuole of the parasite. Consequently, a number of polyaromatic pharmacophores structurally related to clotrimazole have been synthesized with an extension towards clotrimazole-4aminoquinoline conjugates. The antimalarial evaluation of the synthesized scaffolds showed dependence upon some key structural features, viz. (i) the protonatable side chain, (ii) the imidazole ring, and (iii) the aryl/heteroaryl system. The most promising compounds (57, 58 and 59 in Fig. 4) were further evaluated to assess their in vivo efficacy against $P$. chabaudi in CDI mice after oral administration in a 4 day suppression test. The results showed that compounds 57 and 58 have low propensity to produce rapid resistance along with in vivo activity against plasmodia and oral bioavailability.

The above protocol was further extended towards the synthesis of a series of antimalarial hybrids by combining the 4-aminoquinoline unit with that of clotrimazole, as depicted in Fig. $5 .^{74}$ The antimalarial studies were carried out in vitro against CQ-sensitive and -resistant strains as well as in vivo in a rodent malaria model. Compounds $\mathbf{6 3}$ and $\mathbf{6 4}$ displayed strong antimalarial activity against $P$. berghei after oral administration. The compounds have been shown to interfere with the process of heme detoxification, as confirmed by BHIA assay. Cytotoxicity, genotoxic potential and effects on cardiac function of lead compounds were also assessed with the compound $\mathbf{6 3}$ emerging as a good hit because of its promising antimalarial potency and an optimal half-life in mice.

\subsection{4-Aminoquinoline-azithromycin conjugates}

Azithromycin, a semi-synthetic macrolide antibiotic, was recently discovered as a slow acting antimalarial ${ }^{75,76}$ that wields its activity via inhibiting protein synthesis on prokaryote-like ribosomes in $P$. organelle. ${ }^{77}$ Currently, it is in clinical trials and has been used as a combination partner with different

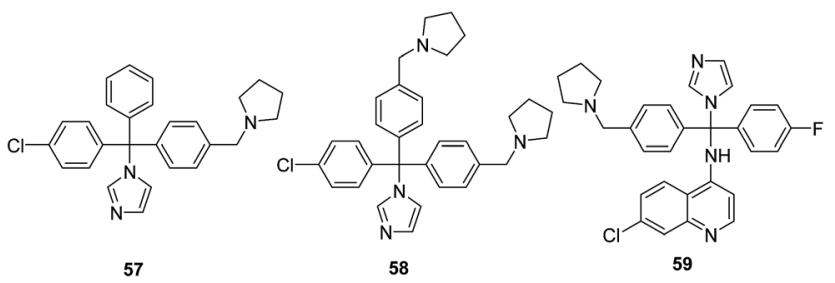

Fig. 4 Most potent compounds 57, 58 and 59. 

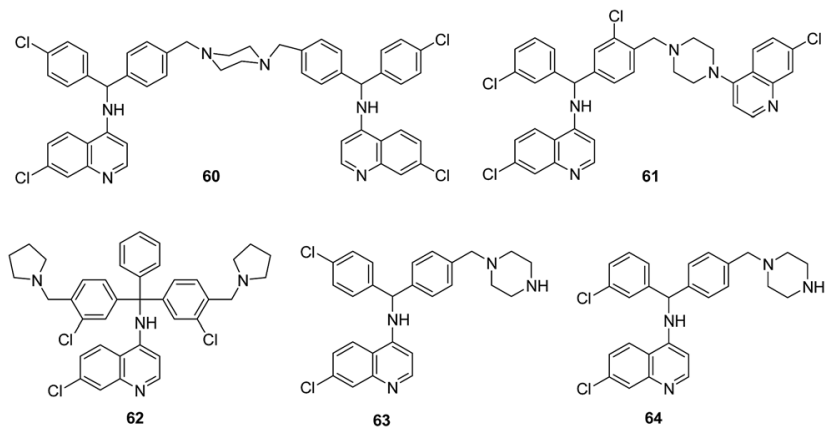

Fig. 5 Molecular conjugates 60,61 and 62 of 4-aminoquinoline and clotrimazole along with their potent precursors 63 and 64 .

antimalarials. Perić and collaborators employed a molecular hybridization strategy for the synthesis of azithromycin-quinoline antimalarial conjugates. ${ }^{7}$ Forty two new azithromycin analogues and azithromycin-quinoline conjugates with amide and amine functionalities were synthesized using simple and economical chemical procedures. These scaffolds displayed 100-fold improvement in in vitro potency with high selectivity, pharmacokinetics (PK) and in vivo efficacy over azithromycin against the $P$. falciparum TM91C235 strain, a multidrug resistant clone from Southeast Asia. The screening of the promising compounds in a mouse efficacy model resulted in the identification of five scaffolds, viz. three amine $(65,66,67)$ and two amide $(68,69)$ derivatives, exhibiting better in vivo efficacy compared to azithromycin (Fig. 6). The most potent of the tested compounds 65 (Fig. 6) showed 100-fold better in vitro activity with excellent pharmacokinetic parameters curing mice more efficiently than azithromycin.

Peric et al. further extended this concept towards the development of the azalide class of antimalarials via synthesis of $2^{\prime}-o-$ substituted-9-deoxo-9 $a$-methyl-9a-aza-9a-homoerythromycin A derivatives covalently linked to varied substituted quinolines at position $2^{\prime}$, as depicted in Fig. $7 .{ }^{79}$ Antimalarial profiles of the test conjugates against the CQ-sensitive 3D7 strain showed 100-fold improvement over azithromycin while 48-fold enhancement was observed against the CQ-resistant W2 strain of $P$. falciparum. These results have facilitated these macrolides for the preclinical development of malaria-specific agents.

\subsection{4-Aminoquinoline- $\beta$-lactam conjugates}

A series of $1 H$-1,2,3-triazole-linked 7-chloroquinoline- $\beta$-lactam conjugates (72 and 73) was synthesized by Kumar and co-workers utilizing click chemistry, as shown in Fig. 8. The antimalarial potency of the synthesized conjugates against a W2-resistant strain of $P$. falciparum was found to be dependent on the substituent present at the $N-1$ position of the $\beta$-lactam ring and the presence of bis-triazole at $\mathrm{C}-3$ position. The observed antimalarial efficacy was further validated by docking studies through inhibition of $P$. falciparum dihydrofolate reductase (PfDHFR). ${ }^{80}$

On the basis of these preliminary results, Kumar et al. further extended their work on 4-aminoquinoline hybridization by synthesizing 4 -aminoquinoline- $\beta$-lactam conjugates linked
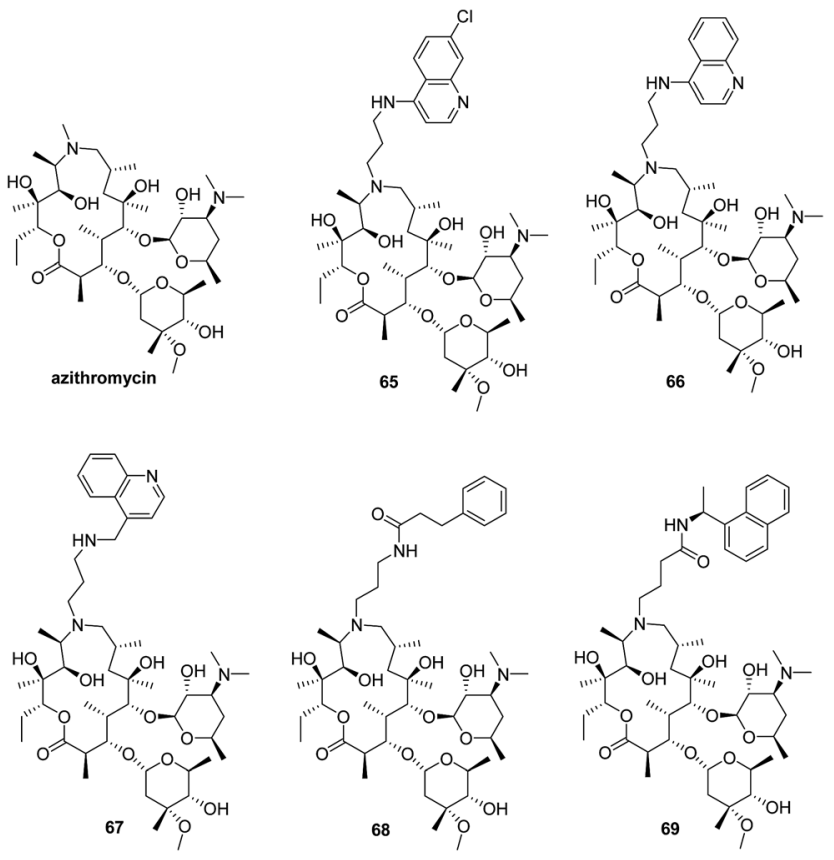

Fig. 6 Structure of azithromycin and potent azalide hybrids used for in vivo study in the mouse model.

via a diverse range of linkers, viz. amide ${ }^{81}(75$, Fig. 8) and nonionizable covalent bonds (74, Fig. 8), ${ }^{81}$ urea $^{82}$ (76, Fig. 8) and oxalamide $^{82}$ (77, Fig. 8) functionalities along with wellmodulated alkyl chain lengths. All the synthesized conjugates were screened for their antimalarial potential against a W2 resistant strain of P. falciparum. Antiplasmodial data revealed that the activity depends on the nature of the linker, the alkyl chain length and the substituent present at the $N-1$ position of the $\beta$-lactam ring. On comparing the potencies among the amide and alkyl chain tethered series, the conjugates linked via non-ionizable covalent alkyl chain linker proved to be better in inhibiting the growth of $P$. falciparum while the introduction of amide functionality adversely affected the activity profiles. The introduction of urea/oxalamide functionalities, on the other hand, resulted in improvement of the antiplasmodial profiles. The most potent and non-cytotoxic conjugate (77a, Fig. 8) among the 7 -chloroquinoline- $\beta$-lactam series with the best

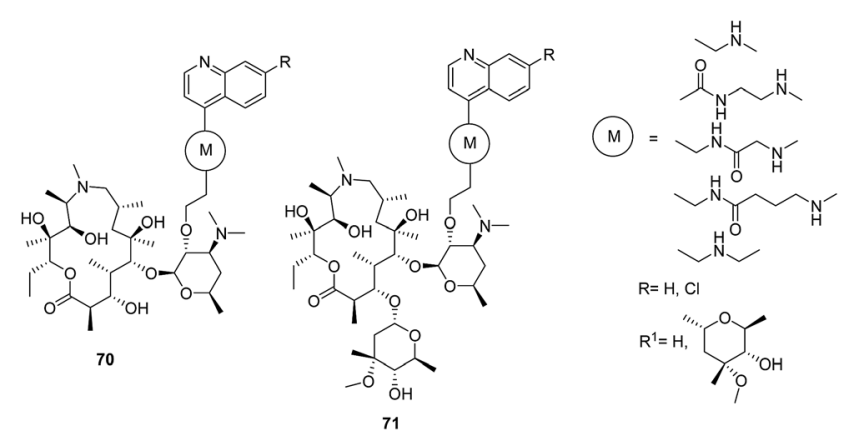

Fig. 7 General structure of hybrids of 2'-O-substituted-9-deoxo-9amethyl-9a-aza-9a-homoerythromycin $\mathrm{A}$ and quinolone. 


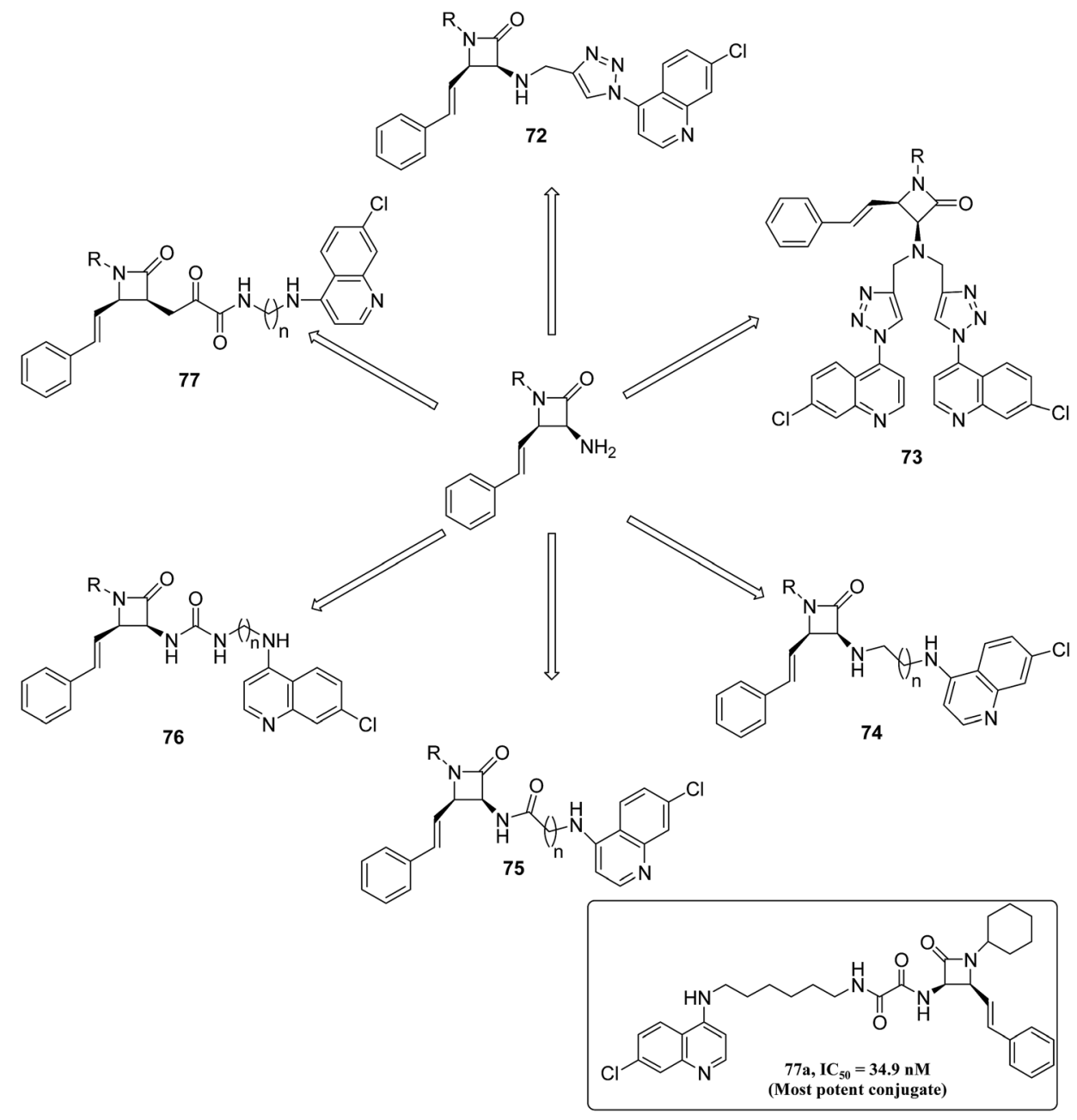

Fig. $8 \beta$-Lactam-4-amino-quinoline conjugates.

combination of $\mathrm{N}$-cyclohexyl substituent at $\mathrm{N}$-1, alkyl chain length $(n=6)$ and oxalamide functionality exhibited an $\mathrm{IC}_{50}$ of $39.9 \mathrm{nM}$.

Further, Kumar et al. synthesized a library of C-3 thiourea tethered $\beta$-lactam-7-chloroquinoline conjugates (78) along with the unexpectedly formed 7-chloroquinoline-thiohydantoin derivatives (79) with the aim of searching antimalarial structure-activity relationships (Fig. 9). The synthesized thioureatethered 7-chloroquinoline- $\beta$-lactam conjugates $\mathbf{7 8}$ were found to be ineffective in inhibiting the growth of $P$. falciparum. However, the unexpected formed product viz. 7-chloroquinoline-thiohydantoins 79 demonstrated nanomolar antimalarial activities against the W2 strain of cultured $P$. falciparum, with the most potent and non-cytotoxic compound exhibiting an $\mathrm{IC}_{50}$ of $39.8 \mathrm{nM}$. $\beta$-Hematin formation studies strongly supported the mechanism of their action via the inhibition of $\beta$-hematin formation having $\mathrm{IC}_{50}$ values comparable to those of $\mathbf{C Q}{ }^{83}$

\subsection{4-Aminoquinoline-isatin conjugates}

Santos and colleagues designed, synthesized and evaluated 3-methylene-substituted indolinones (Fig. 10, 80-85) as falcipain inhibitors and antiplasmodial agents. ${ }^{84}$ Various indolinones with a Leu-i-amyl recognition moiety were shown to display fair inhibitory activity of $P$. falciparum cysteine protease falcipain-2 and antimalarial potency against the W2 strain of $P$. falciparum in the low $\mu \mathrm{M}$ range. Importantly, compounds lacking the recognition moiety were devoid of FP-2 inhibition and interaction of the Leu-i-amyl sequence with the S2 pocket. Further, the introduction of a 4-aminoquinoline to the C-3 position of the indolinone-2-one scaffold (85) led to a considerable enhancement in the antiplasmodial activity, suggestive of the fact that the 3-methylene-indolinone-2-ones could become forthcoming lead compounds for the development of new antimalarials.

Kumar et al. synthesized a library of isatin-7-chloroquinoline conjugates linked by $1 H$-1,2,3-triazole moiety along with assessment of their antiplasmodial profiles (Scheme 15). The tested compounds (88) were devoid of antiplasmodial activity, while 89, having an alkyl chain between isatin and 7-chloroquinoline moieties, displayed enhancement in antiplasmodial potency. Activity was found to depend on the C-5 substituent of the isatin ring and on the alkyl chain length between the isatin and 7-chloroquinoline moieties. The most potent conjugate, 


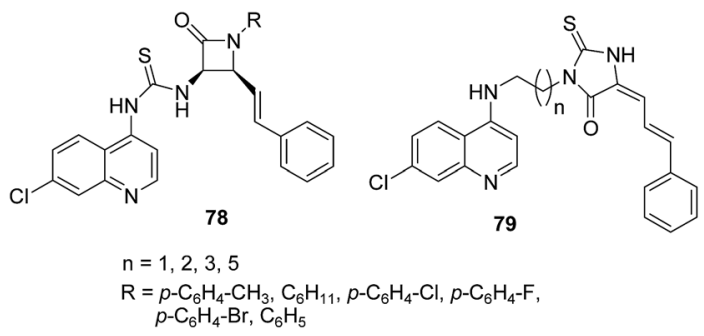

Fig. 9 General structures of $\beta$-lactam-7-chloroquinoline conjugates (78) and 7-chloroquinoline-thiohydantoin derivatives (79).

with the best combination of propyl linker $(n=3)$ and chlorosubstituent at the C-5 position of isatin ring, exhibited an $\mathrm{IC}_{50}$ value of $1.21 \mu \mathrm{M}^{85}$

The above work was further extended towards the synthesis of triazole linked isatin-7-chloroquinoline (93) and 3-hydroxyindole-7-chloroquinoline hybrids (94) by following the synthetic protocol shown in Fig. 11 along with their antiplasmodial evaluation against the CQ-resistant W2 strain. $^{86}$ studies revealed the dependence of activities on the length of alkyl chain but independent of the nature of the substituent present at the $\mathrm{C}-5$ position of the isatin or indole ring. Most of the conjugates were less active than the standard drug CQ; however, the best conjugate with an optimum combination of 3-hydroxy-indole ring and $n$-butyl linker exhibited an $\mathrm{IC}_{50}$ value of $69 \mathrm{nM}$, comparable to that of CQ. Further, Kumar and co-workers synthesized piperazine tethered 7-chloroquinolineisatin conjugates (95 and 96, Fig. 12) by both direct nucleophilic substitution and $\mathrm{Cu}(\mathrm{I}) \mathrm{Cl}$ mediated Mannich reaction. ${ }^{87}$ All the synthesized conjugates were assessed for their anti-plasmodial, anti-tubercular and cytotoxic evaluation. Analysis of antiplasmodial activity data against the W2 strain showed that none of conjugates were as active as $\mathbf{C Q}$, although some conjugates proved to have good anti-malarial efficacy with $\mathrm{IC}_{50 \mathrm{~s}}$ ranging from 0.22 to $0.27 \mu \mathrm{M}$.

Anti-tubercular evaluation against M. tuberculosis revealed the dependence of the activity profile on the presence of a substituent at the $\mathrm{C}-5$ position of the isatin ring whereas longer alkyl chain lengths badly affected the efficacy of the compounds. Compound 95, with a fluoro substituent, was shown to have good anti-TB efficacy with six times more potency than the standard drug, Cephalexin. On the other hand, Mannich adducts (96) were found to be ineffective in inhibiting the growth of M. tuberculosis. The most potent compound 95a (Fig. 12), with an $\mathrm{IC}_{50}$ of $0.22 \mu \mathrm{M}$ against the CQ-resistant strain of P. falciparum and $31.62 \mu \mathrm{M}$ against the $3 \mathrm{~T} 6$ cell line, exhibited a high selectivity index.

Recently, Kumar et al. synthesized $\beta$-amino alcohol tethered isatin-4-aminoquinoline conjugates with the aim of investigating their antimalarial structure activity relationship. SAR studies have shown a clear preference for shorter alkyl chain lengths $(n=2,3)$ and the presence of a Cl-substituent at the C-5 position of the isatin ring for good activity. The two most potent and non-cytotoxic conjugates (97 and 98, Fig. 13) displayed antiplasmodial potency comparable to that of $\mathbf{C Q}$, with $\mathrm{IC}_{50}$

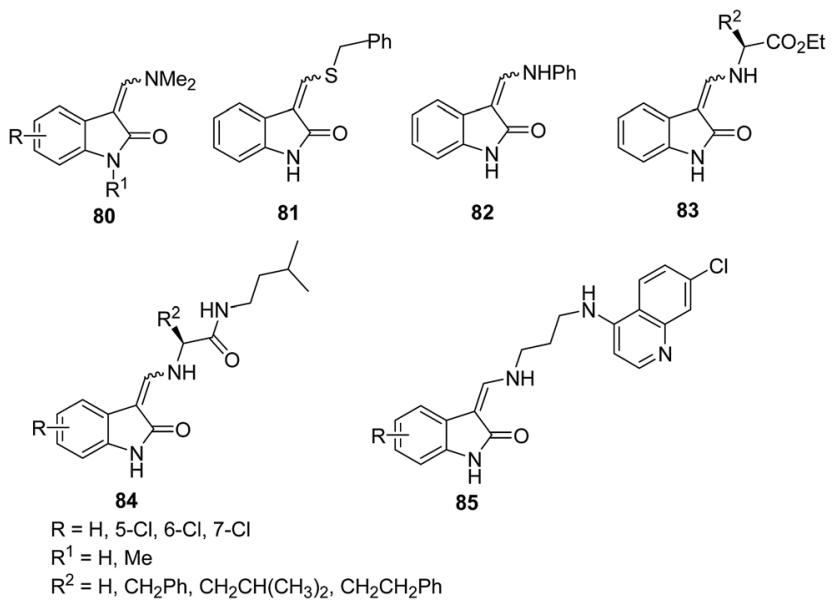

Fig. 10 General structures of synthesized 3-methylene-substituted indolinone analogues 80-85.

values of 11.7 and $13.5 \mathrm{nM}$, against the $\mathrm{W} 2$ resistant strain of P. falciparum. ${ }^{88}$

\subsection{Organometallic based 4-aminoquinoline conjugates}

Over the past few years, bio-organometallic chemistry has emerged as a quickly growing and maturing area that associates organometallic chemistry to medicine, biology and molecular biotechnology ${ }^{89}$ The replacement of organic ligands with metal complexes has the ability to enhance the activity of biological compounds along with potential advantages, such as the synthesis of stable metal complexes with predictable structures, the capability to tune ligand affinities in accordance with their electron transfer properties, substitution rates and reduction potentials along with effective biological targeting..$^{90}$ Among the implausible number and variety of roles that metals play in current medicine, cancer $^{91}$ and malaria ${ }^{92}$ treatments are appreciated as possibly the most important application of metal-based drugs.

In malaria chemotherapy, resistance to established drugs may be overcome by bio-organometallics through new metal specific modes of action. Ferroquine (99, FQ, SSR97193, Fig. 14), the ferrocene analogue of $\mathbf{C Q}$, is the most pertinent example of a bio-organometallic compound that showed higher antimalarial

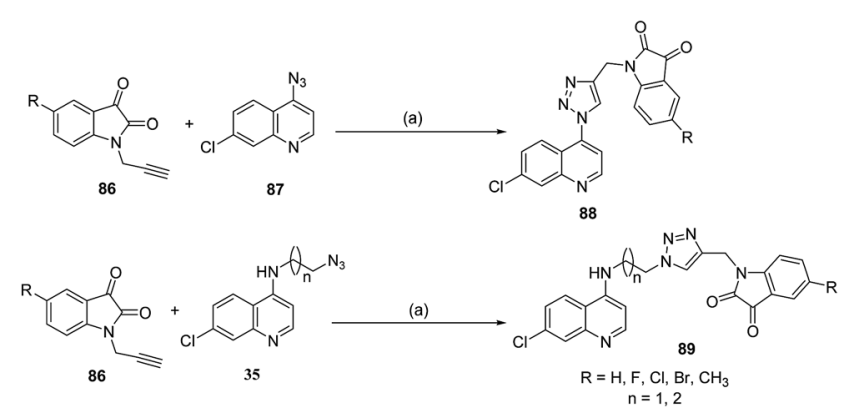

Scheme 15 Reagents and conditions: (a) $\mathrm{CuSO}_{4}$, sodium ascorbate, $\mathrm{EtOH}: \mathrm{H}_{2} \mathrm{O}$, rt $7 \mathrm{~h}$. 


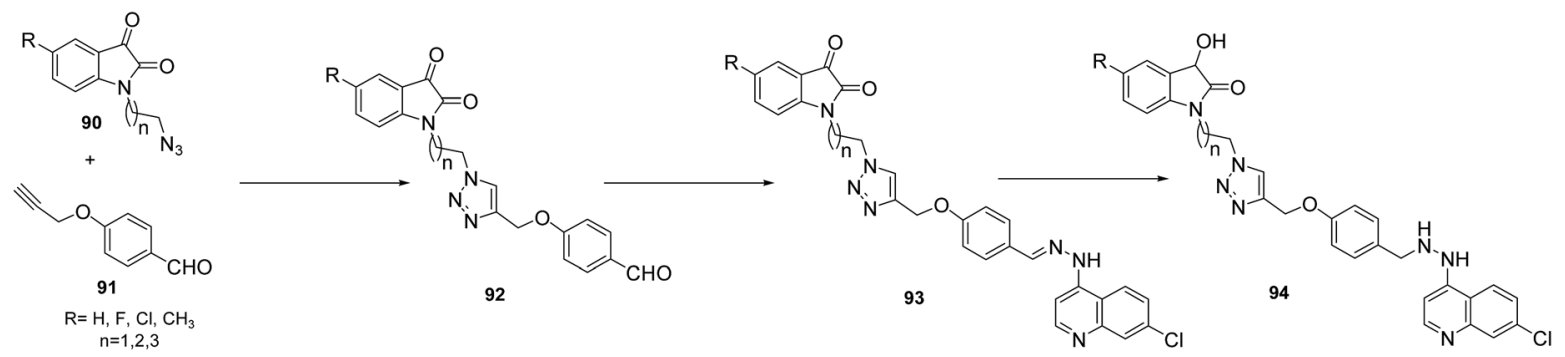

Fig. 11 General structures of target hybrid compounds.

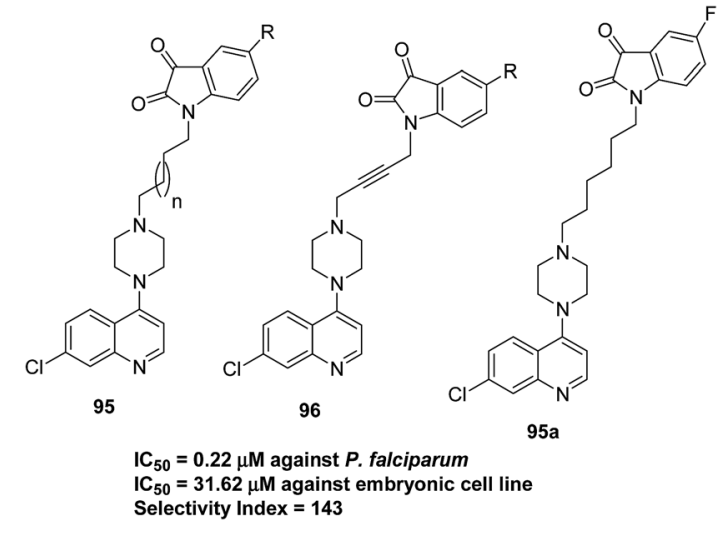

Fig. 12 General structures of piperazine tethered 7-chloroquinolineisatin conjugates 95 and 96 along with most potent conjugate 95a.
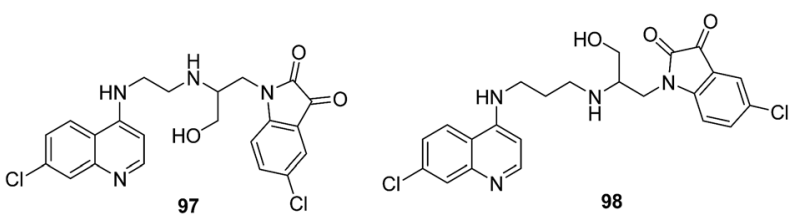

Fig. 13 The two most potent $\beta$-amino alcohol tethered isatin-4aminoquinoline conjugates 97 and 98 .

potency in vivo on mice infected with $P$. yoelii NS and $P$. berghei $\mathrm{N}$. It is twenty two times more active against schizontocides than CQ against the CQ-resistant strain of $P$. falciparum. The effectiveness of FQ against the CQ-resistant strain could possibly be due to its capability to penetrate in the infected cells. $^{93}$

Biot et al. further utilized the ferroquine pharmacophore as a template for the design of two novel series of ferrocenic antimalarial conjugates comprising FQ derivatives hybridized with

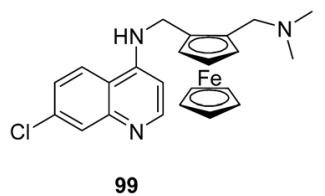

Fig. 14 Structure of ferroquine (FQ), 99. a glutathione reductase inhibitor (or glutathione depletor) via an appropriate cleavable amide bond (Scheme 16). In vitro antimalarial studies on CQ-susceptible (NF54) and CQ-resistant (K1) strains were conducted, with emphasis on the study of their mechanism of action. ${ }^{94}$ Among the series of synthesized conjugates, the compounds 101a-101f were found to exhibit the most potent in vitro activity with $\mathrm{IC}_{50}$ values in the nanomolar range. However, their antiplasmodial activity was slightly less than the precursor FQs 100a-100f possibly due to the cleavage of the amide bond and the oxidative metabolism of the side chain of the FQ in the digestive vacuole of the parasite. The observed antiplasmodial activity of FQ was mainly attributed to the high potential of the ferrocene core exhibiting a redox mechanism different from the mechanism of action of chloroquine.

The discovery of ferroquine (99, FQ, SSR97193) as a promising antimalarial prompted the synthesis of corresponding rhenium organometallics to measure the significance of the ferrocenyl group (Scheme 17). ${ }^{95}$ All the synthesized compounds (organic along with their organometallic counterparts) were assessed for their in vitro antiplasmodial potency against the CQ-resistant (W2) and the CQ-susceptible (3D7) strains of $P$. falciparum. The cyrhetrene conjugates (105 and 106), however, were found to be less active than the corresponding ferrocene and organic analogues.

Orvig et al. explored the synthesis of CQ-bridged ferrocenophane analogues of ferroquine in which the terminal nitrogen

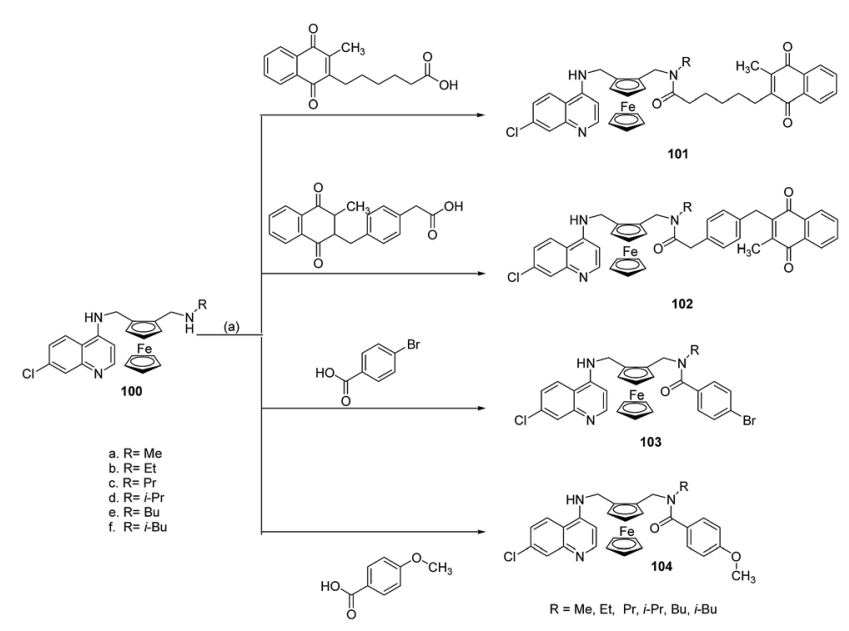

Scheme 16 Reagents and conditions: (a) EDCl, DCM, rt. 

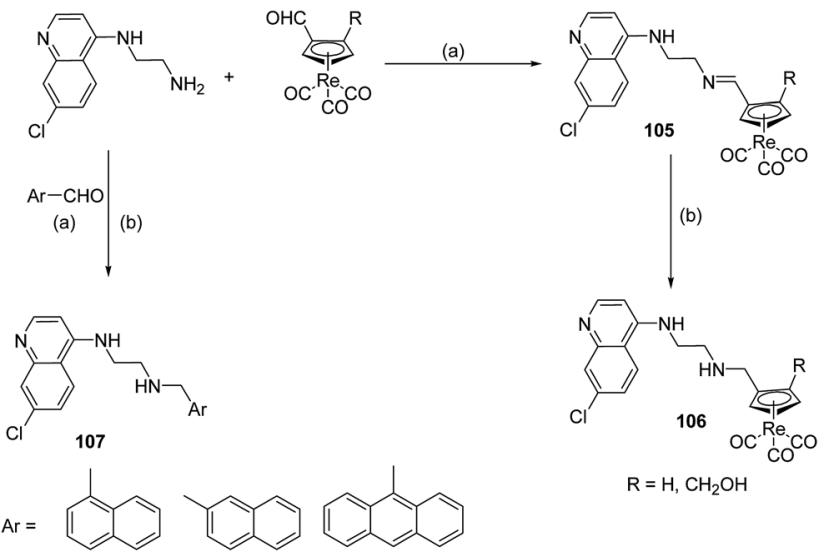

Scheme 17 Reagents and conditions: (a) $\mathrm{MeOH}$, rt; (b) $\mathrm{NaBH}_{4}$, $\mathrm{MeOH}$, rt.

of the CQ derivative bridged the two cyclopentadienyl rings of ferrocene (110a-110e) alongside their mono-substituted ferrocene analogues (109a-109e) and organic fragments (108a-108e) (Fig. 15). ${ }^{96}$ All the synthesized compounds were screened for their antiplasmodial potency and were found to be active over both CQ-sensitive and -resistant strains. Mono-substituted ferrocenyl analogues were found to be more active than the CQ-bridged ferrocenyl derivatives while the later compounds, viz. 110a-110e, retained their activity against the CQ-resistant strains. SAR studies revealed the loss of activity with increased branching while the length of alkyl spacer did not seem to influence their antiplasmodial profiles. The physical properties, viz. the presence or absence of hydrogen bonding interactions, the degree of rigidity and the lipophilicity of these conjugates, were correlated to their biological activity. A balance between lipophilicity and hydrophilicity seemed to influence the biological profile of the conjugates while the structural characteristics, such as conformation and size, were found to be essential to overcoming the drug resistance.

The methodology was further extended with the synthesis of a new class of organometallic antimalarials comprising a ferrocene nucleus tethered to a CQ analogue and a 1,2,3,5(diisopropylidene)- $\alpha$-D-glucofuranose moiety in a $1,1^{\prime}$-heteroannular substitution pattern. Synthesis of these compounds was facilitated by orthogonal functionalization of ferrocene resulting in 1-acetoxy-1'-(1,3-dioxan-2-yl)ferrocene as a key intermediate for the modular introduction of the carbohydrate. This was followed by reductive amination with CQ derivatives, yielding 1-[3-(7-chloroquinolin-4-ylamino)alkylamino]-1'-[6(1,2;3,5-diisopropylidene)- $\alpha$-D-glucofuranosidyl]ferrocenes 117119 (Fig. 16). These trifunctional hybrids were assessed for their antiplasmodial activity against CQ-susceptible (D10) and CQ-resistant (Dd2 and K1) strains of $P$. falciparum. ${ }^{97}$ No correlation could be established between either the strength of intramolecular H-bonding or the polar surface area and antimalarial potential of these compounds. Antiplasmodial studies indicated that the activity of the synthesized compounds (117-119) showed improvement in comparison to their either 1,2-disubstituted regio-isomers (114-116) or to

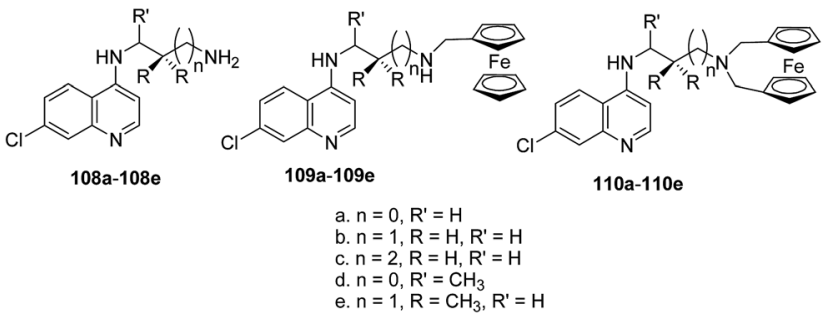

Fig. $15 C Q$ derivatives 108a-108e, mono-substituted CQ-ferrocenyl compounds $109 \mathrm{a}-109 \mathrm{e}$, and 1,1'-disubstituted bridged $\mathrm{CQ}$-ferrocenyl hybrids $110 \mathrm{a}-110 \mathrm{e}$.

monosubstituted ferrochloroquines (111-113). The side chain length linking CQ with the ferrocene molecule did not have any effect on the observed activity.

After the discovery of FQ as a potential antimalarial drug, an extensive study was carried out to explore its derivatives with better activity, enhanced ADME properties and structure activity relationship. In a recent study, 4-aminoquinoline, trioxane and ferrocene were covalently linked to form a hybrid (120, Fig. 17) with the aim of studying its antimalarial efficacy. The scaffold revealed potent activity against CQ-resistant $P$. falciparum and was further evaluated in vivo against $P$. vinckei petteri infected mice. In vivo studies showed a marked decrease in the parasitemia to a lower than detectable level. ${ }^{98}$ Two new "half sandwich" ( $\eta^{6}$-arenequinoline $) \mathrm{Cr}(\mathrm{CO})_{3}$ complexes, viz. $\left[\eta^{6}-N-(7-\right.$ chloroquinolin-4-yl)- $N^{\prime}$-(2-dimethylaminomethylbenzyl)ethane1,2-diamine $]$ tricarbonylchromium and $\left[\eta^{6}-N\right.$-(7-chloroquinolin4-yl)- $N^{\prime}$-(2-dimethylaminobenzyl)-ethane-1,2-diamine]tricarbonylchromium compounds (121 and 122, Fig. 17) were prepared and tested in vitro for their antiplasmodial efficacy against CQ-susceptible and CQ-resistant strains. The efficacy of complex 122 was twofold higher compared to the organic ligand. ${ }^{99}$ In search of new efficient antimalarial complexes, Smith et al. synthesized a series of poly(propylene-imine) dendrimers functionalized with ferrocenyl thiosemicarbazones hybridized to the periphery of the molecule (123, Fig. 17). These metallodendrimers exhibited antiplasmodial activity in the low $\mu \mathrm{M}$ range against the W2 strain of $P$. falciparum. Their antiplasmodial potencies were better than non-conjugated ferrocenyl thioesters as well as the free dendritic ligand. ${ }^{\mathbf{1 0 0}}$ Further, organometallic complexes, viz. cymantrene $\left(\mathrm{CpMn}(\mathrm{CO})_{3}\right)$ and cyrhetrene
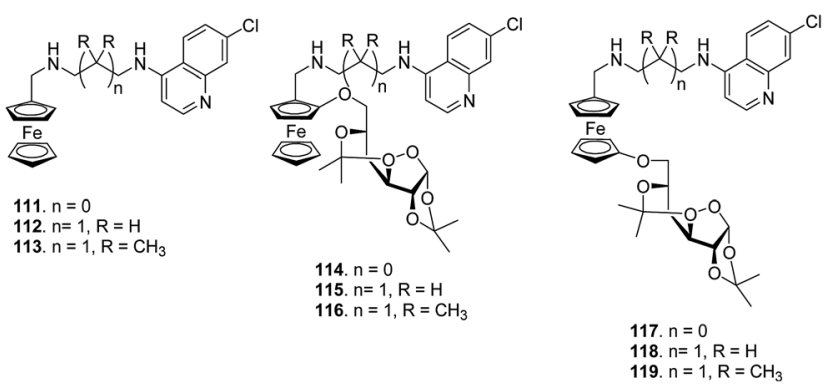

Fig. 16 Mono-, 1,2-disubstituted, 1,1'-disubstituted ferrocenyl chloroquine glucofuranose conjugates 111-113, 114-116 and 117-119. 


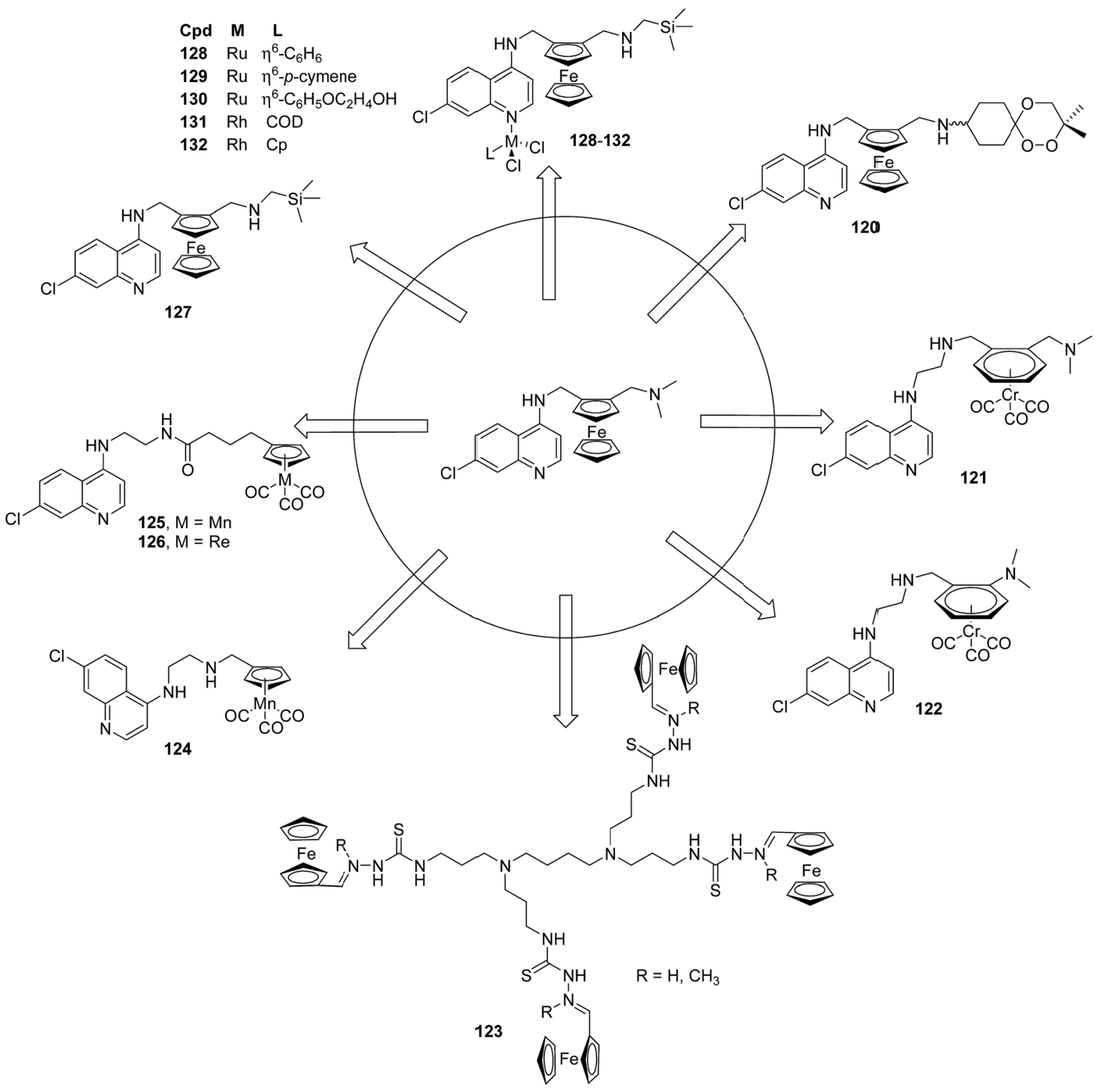

Fig. 17 Some conjugates of ferroquine.

$\left(\mathrm{CpRe}(\mathrm{CO})_{3}\right)$ tethered with 4-aminoquinolines, were reported (124-126, Fig. 17) and bio-evaluated for their malarial potency against D10 and Dd2 strains of $P$. falciparum. The low resistance indices (RI) of these complexes indicated that these complexes of manganese and ruthenium may act as a lead for further development of new antimalarial compounds. ${ }^{101}$

Smith et al. in a recent communication synthesized a new carbosilane congener of ferroquine (127, Fig. 17) by including an organosilicon moiety in the side chain of ferroquine. The carbosilane congener was further utilized for the synthesis of a corresponding series of metal complexes of neutral heterometallic ruthenium and rhodium (128-132), as shown in Fig. 17. All the newly synthesized compounds were evaluated for antiplasmodial activity against the NF54 and Dd2 strains of $P$. falciparum. The presence of the trimethylsilyl group in compound 127 exhibited superior activity $\left(\mathrm{IC}_{50}=7.32 \mathrm{nM}\right)$ as compared to ferroquine $\left(\mathrm{IC}_{50}=42.65 \mathrm{nM}\right.$ ) against the NF54 strain. Among synthesized complexes, $\mathbf{1 3 0}$ was the most active with $\mathrm{IC}_{50}$ values of 4.86 and $35.91 \mathrm{nM}$ against NF54 and Dd2 strains, respectively. ${ }^{102}$

Kumar and co-workers reported the synthesis of a gallium(III) complex with 7-chloroquinoline thiosemicarbazone as a ligand with antimalarial efficacy against 3D7 strain of $P$. falciparum (Scheme 18). The synthesized complex 134 exhibited improved antimalarial activity than lumefantrine on 3D7 isolate of $P$. falciparum. Further, the synthesized complex 134 was evaluated for anti-proliferative activity against different cancer cell lines and complex proved to be 31 times more potent on colon cancer cell line HCT-116 compared with the standard drug etoposide with considerably less cytotoxicity on non-cancerous colon fibroblast CCD-18Co. ${ }^{103}$ 


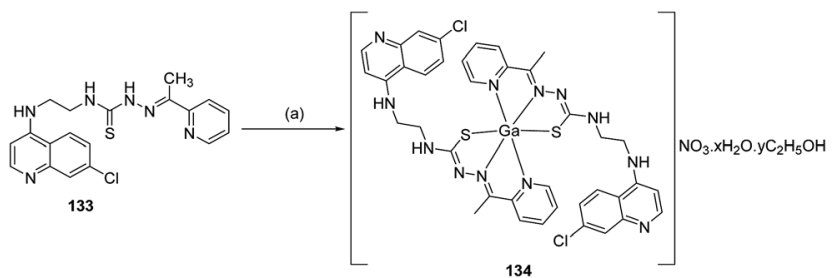

Scheme 18 Reagents and conditions: (a) $\mathrm{Ga}(\mathrm{NO})_{3} \cdot \mathrm{H}_{2} \mathrm{O}$, ethanol, reflux, $2 \mathrm{~h}$

\subsection{Artemisinin and trioxaquine based 4-aminoquinoline conjugates}

The benefits of molecular hybridization over combination therapy can be well rationalized by the report of Walsh and coworkers involving the synthesis of dihydroartemisinin-quinine hybrids (139). The compound was prepared by following a sequence of steps as shown in Scheme 19 involving an initial protection of quinine with TBDMSiCl with subsequent oxidation to form carboxylic acid and coupling with dihydroartemisinin. ${ }^{45}$ The antimalarial efficacy of the synthesized hybrids was determined against CQ-sensitive 3D7 and CQ-resistant FcB1 strains of $P$. falciparum. The conjugate proved to be more potent than either quinine or dihydroartemisinin while a three-fold improvement in efficacy was observed over a $1: 1$ mixture of quinine and dihydroartemisinin.

Rationalization of literature has revealed that the variation in the length of alkyl chain between two nitrogen atoms of the 4-diaminoalkyl side chain has a marked influence on the antimalarial efficacy. The compounds with either smaller alkyl chain length $(n=2-4)$ or with longer alkyl chain length $(n=$ 10-12) retained their activities against CQ-resistant strains,

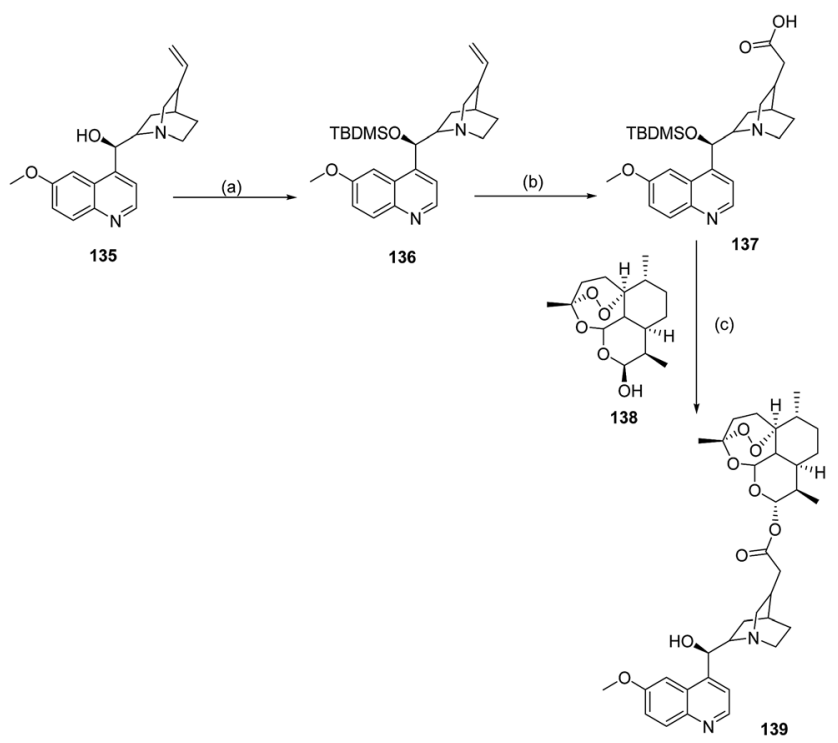

Scheme 19 Reagents and conditions: (a) TBDMSCl, Et ${ }_{3} \mathrm{~N}, \mathrm{DMAP}, \mathrm{DMF}$, rt; (b) (i) $\mathrm{BH}_{3}-\mathrm{THF}$, diglyme, $0{ }^{\circ} \mathrm{C}$; (ii) $\mathrm{Me}_{3} \mathrm{NO} \cdot 2 \mathrm{H}_{2} \mathrm{O}, 100{ }^{\circ} \mathrm{C}$; (iii) Jones reagent, acetone, rt; (c) (i) 2,6-dichlorobenzoyl chloride, $\mathrm{Et}_{3} \mathrm{~N}$, DMAP, DCM, rt; (ii) TBAF, THF, rt. whereas the compounds with chain length $n=5-8$ showed a relative decrease in the activity. ${ }^{\mathbf{1 0 4}}$ Chibale and co-workers exploited a molecular hybridization protocol for the preparation of dihydroartemisinin-7-chloroquinoline conjugates (141 and 143) prepared via aza-Michael addition reaction along with the analogues of dihydroartemisinin (DHA) derivatives 142 (Scheme 20). These were tested for their antimalarial profiles against CQ-sensitive (D10) and CQ-resistant (Dd2) strains of $P$. falciparum. The length of alkyl chain introduced as the spacer was chosen to be between 2 and 6 carbon atoms for optimal biological activity. ${ }^{105}$ The evaluation data revealed a substantial increase in antimalarial efficacy of the hybrids over the precursors 7 or 28 while a decrease in antimalarial efficacy was observed with the increase in chain length. DHA derivatives prepared via aza-Michael addition with available amines were found to be more effective in the growth inhibition of $P$. falciparum suggestive of the fact that the DHA nucleus is mainly responsible for the observed antimalarial activity of the hybrids. The target compound $\mathbf{1 4 1}$ and three dihydroartemisinin derivatives of 125 showed excellent antiplasmodial activity with $\mathrm{IC}_{50}$ values of $\leq 10 \mathrm{nM}$ against both D10 and Dd2 strains of P. falciparum and low cytotoxicity against human cell lines (HeLa cells).

N'Da further extended his work on the synthesis of artemisinin-quinolines and their hybrid-dimers from dihydroartemisinin and aminoquinolines (Scheme 21). ${ }^{\mathbf{1 0 6}}$ The synthesized hybrids (145) obtained as $\beta$-isomers were assessed for their antiplasmodial activity against CQ-sensitive and -resistant strains of $P$. falciparum along with cytotoxicity profiles against a mammalian cell-line. Although none of the hybrids was found to be more potent than DHA, two were more active than CQ. A hybrid-dimer with an optimum chain length of three carbon atoms displayed excellent antimalarial potency, with $\mathrm{IC}_{50}$ values of 5.31 and $28.43 \mathrm{nM}$ against D10 and Dd2 strains of P. falciparum.

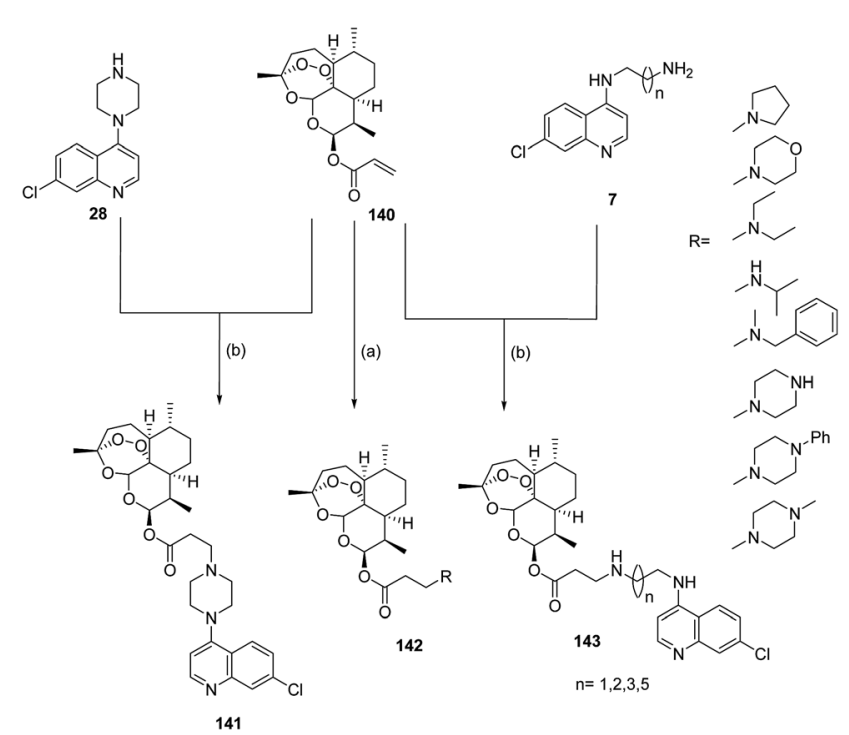

Scheme 20 Reagents and conditions: (a) appropriate amine, DBU, $\mathrm{CH}_{3} \mathrm{CN}, \mathrm{rt}, 12 \mathrm{~h}$; (b) DBU, DMF, $\mathrm{N}_{2}, \mathrm{rt}, 12 \mathrm{~h}$. 

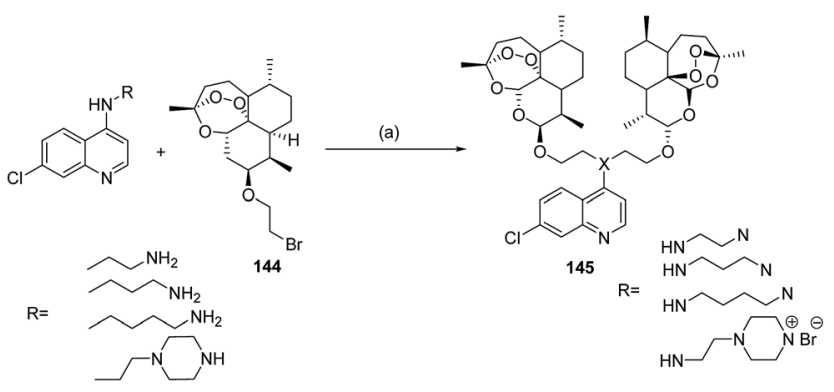

Scheme 21 Reagents and conditions: (a) DMF, $90-110^{\circ} \mathrm{C}, 6-8 \mathrm{~h}$.

N'Da with his co-workers explored the synthesis of dihydroartemisinin-4-aminoquinoline conjugates (146, 147 and 148) with their subsequent conversion to oxalate salts (Scheme 22). In vitro antimalarial activity was tested against CQ-sensitive D10 and -resistant Dd2 strains of $P$. falciparum while cytotoxicity was determined against mammalian Chinese Hamster Ovarian (CHO) via 3-(4,5-dimethylthiazol-2-yl)-2,5-diphenyltetrazoliumbromide (MTT)-assay. ${ }^{107}$ Most of the synthesized conjugates showed comparable to higher activity to that of the standard drug CQ with $\mathrm{IC}_{50 \mathrm{~s}}$ ranging from 17.2 to $38.9 \mathrm{nM}$. The oxalate salts proved to be seven times more potent than the standard drug CQ against the CQ-resistant strain of $P$. falciparium.

In 2011, Chibale et al. extended the synthesis of artemisinin4-aminoquinoline conjugates $(\mathbf{1 5 1}, \mathbf{1 5 2})$ via Ugi four-component condensation (Ugi-4CR) reaction (Scheme 23) ${ }^{\mathbf{1 0 8}}$ using paraformaldehyde as aldehyde component, 7-chloro-4diaminoalkylquinoline as the amine input, artelinic acid/1,4napthoquinone acid as acid input, and cyclohexyl-or tert-butyl isocyanide as the isocyanide input. Antiplasmodial studies of the synthesized conjugates showed comparable potency against CQ-resistant K1 and CQ-sensitive D10 strains of P. falciparum.

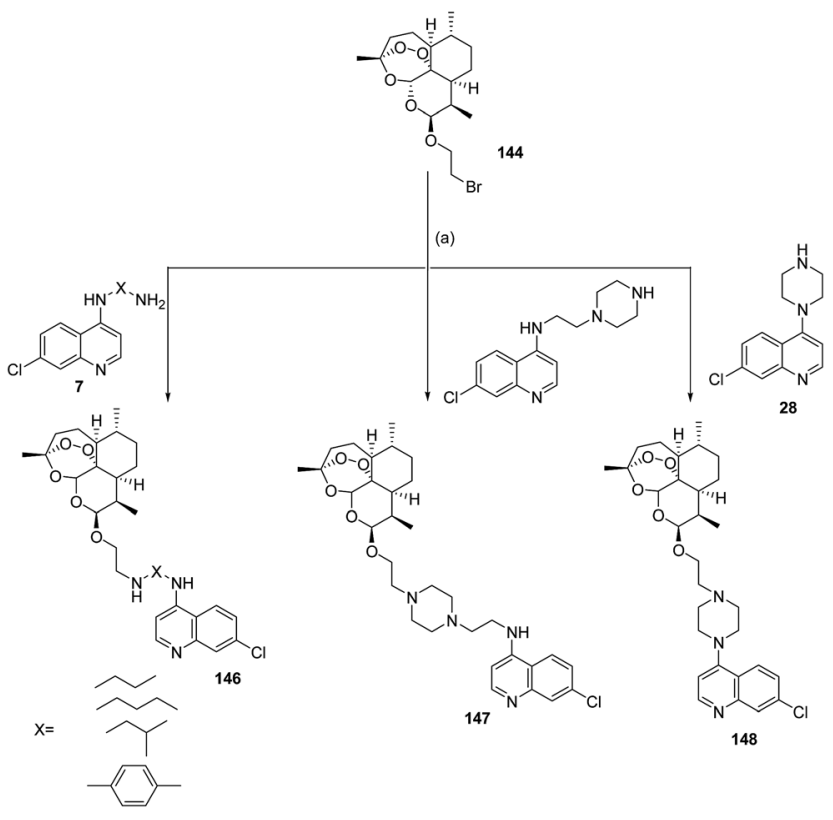

Scheme 22 Reagents and conditions: (a) DMF, $70-80{ }^{\circ} \mathrm{C}, 4-6 \mathrm{~h}$.
Mechanistically, the conjugates exhibited potent inhibitory activities of $\beta$-hematin formation and appeared to inhibit endocytosis as evident by the decrease in the number of transport vesicles in the parasite.

Meunier et al. reported the synthesis of trioxaquines 154 as potential antimalarials by following the synthetic protocol as depicted in Scheme 24. ${ }^{109}$ Compound trioxaquine (PA1103/ SAR116242) 154, obtained from this study, proved to be highly active against several sensitive and resistant strains of $P$. falciparum at $\mathrm{nM}$ concentrations. It has also shown activity on multidrug-resistant strains attained from fresh patient isolates in Gabon along with good drug profiles viz. absorption, metabolism and safety parameters. Efficacy of this conjugate via oral route is very high with total curing of mice infected with CQsensitive and -resistant strains of Plasmodium at $26-32 \mathrm{mg} \mathrm{kg}^{-1}$.

The selection of trioxaquine (PA1103/SAR116242) for antimalarial drug development strongly confirms the role of molecular hybridization in furnishing molecules with satisfactory pharmacological and safety profiles to allow regulatory drug development. Guided by similar rationale, O'Neill and coworkers reported the synthesis of semi-synthetic trioxaquines and trioxolaquines in excellent yields and assessed their antimalarial activities against CQ-sensitive (3D7) and -resistant (K1) strain of $P$. falciparum. Compounds of both the series showed activity in low nM range with some of the analogues being more active than artemisinin. The structure of two of the most active compounds 155 and 156 of the series is as shown in Fig. 18. ${ }^{\mathbf{1 1 0}}$

\subsection{Miscellaneous antimalarial 4-aminoquinoline conjugates}

A library of tetrahydro- $1 H$ - $\beta$-carbolines-4-aminoquinoline hybrids 159 were identified as a novel class of highly potent antimalarial agents by Chauhan et al. using molecular hybridization approach with MIC values ranging from 0.05 to $19.08 \mu \mathrm{M}$ against CQ-sensitive strain NF-54 of $P$. falciparum (Scheme 25). ${ }^{111}$ Most potent of the synthesized conjugate was

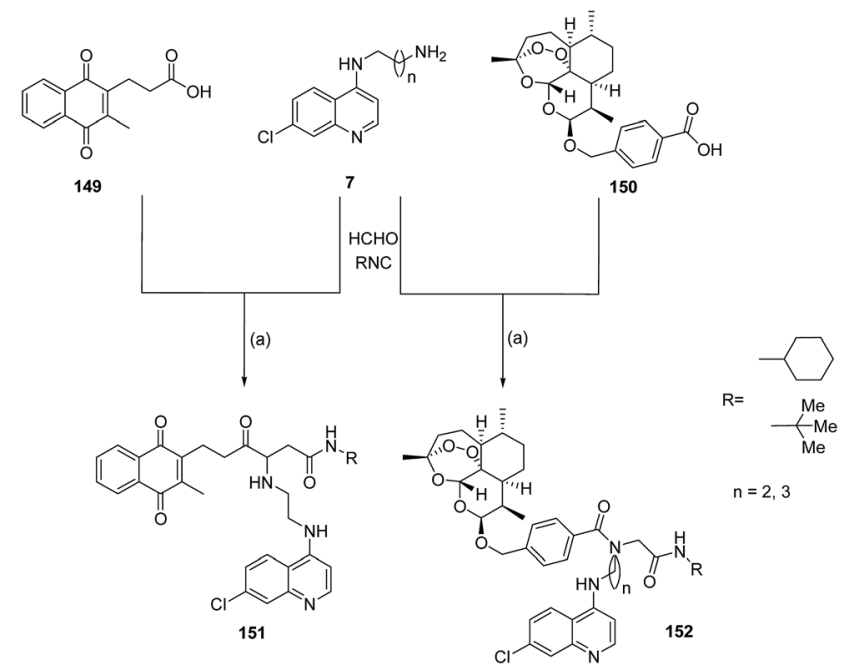

Scheme 23 Reagents and conditions: (a) succinic acid, silver nitrate, ammonium persulfate, $30 \%$ aq. $\mathrm{CH}_{3} \mathrm{NH}_{2}, 65-70{ }^{\circ} \mathrm{C}, 3 \mathrm{~h}$. 


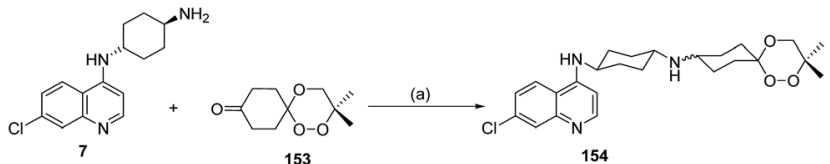

Scheme 24 Reagents and conditions: (a) $\mathrm{NaBH}_{3} \mathrm{CN}, \mathrm{MeOH}, \mathrm{HCl} / \mathrm{i}-$ $\mathrm{PrOH}$.

found to have seven folds more activity than the standard drug CQ and therefore warrants the development of these hybrids in antimalarial chemotherapy.

In 2011, Egan and collaborators proposed the synthesis of dibemethin-coupled 4-aminoquinolines $\mathbf{1 6 2}$ as potential antimalarial agents with a view to obtain novel hybrids capable of inhibition of hemozoin formation in the digestive vacuole of parasite (Scheme 26). ${ }^{112}$ These hybrids were equally potent against cultured CQ-sensitive (D10) and CQ-resistant (K1) $P$. falciparum. Several compounds exhibited in vitro antimalarial potency below $100 \mathrm{nM}$ against both strains of the parasite while none of compound exhibited cross-resistance with the standard drug CQ. The compounds with promising in vitro activity and non-cytotoxicity against mammalian cell line were screened for their in vivo activity against $P$. berghei. The active compounds exhibited significant in vivo activity and reduces parasitemia by over $99 \%$. SAR studies showed marked dependence of activity on the site of attachment of 4-aminoquinoline with side chain of dibemethin as well as the substituent on the terminal phenyl ring of dibemethin. Researchers further investigated the interaction of this series of compounds with $\mathbf{C Q}$ transport by making use of the Xenopus oocyte expression system, which directly measures CQ transport by P. falciparum CQ-resistance transporter (PfCRT) and its inhibition with potential resistancereversers or reversed-CQ compounds. The results of this study confirmed 4-aminoquinoline-dibemethin hybrids as the first example of reversed-CQ antimalarials with capability to inhibit both PfCRT and hemozoin formation.

Recently, Chibale et al. reported the design and synthesis of novel $\beta$-amino alcohol derivatives of 4 -aminoquinoline (164) with an extension to 4-aminoquinoline-oxazolidinone conjugates (165, Scheme 27). ${ }^{113}$ In vitro antimalarial activity was evaluated against CQ-sensitive (3D7) and CQ-resistant (K1) strains of $P$. falciparum. Few of the $\beta$-amino alcohol analogues

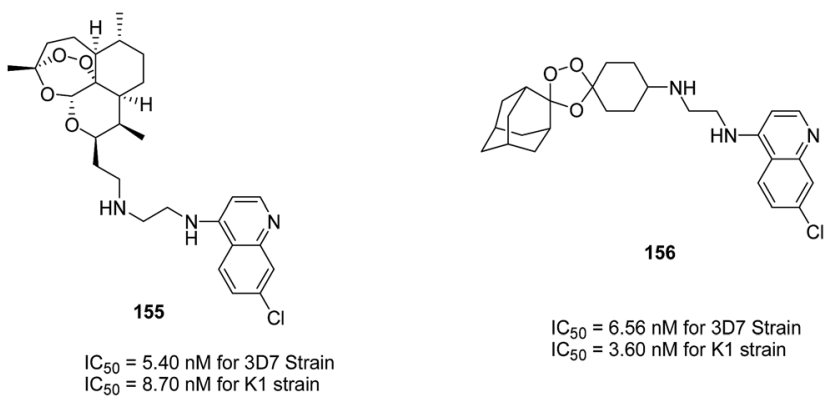

Fig. 18 Most potent 1,2,4-trioxaquines and 1,2,4-trioxolaquines conjugates.
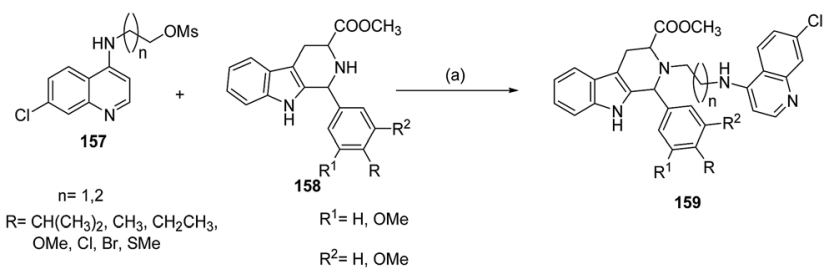

Scheme 25 Reagents and conditions: (a) DMF, $120^{\circ} \mathrm{C}$, high pressure.

were more active compared to $\mathbf{C Q}$ against the $\mathbf{C Q}$-sensitive strain while the potency decreased considerably against the CQ-resistant strain, revealing cross resistance of these conjugates with CQ. The conversion of $\beta$-amino alcohol to oxazolidinones by reacting with tris-phosgene resulted in a considerable decrease in the activities of the synthesized conjugates against both CQ-sensitive and CQ-resistant strains.

Chauhan et al. synthesized sixteen new 4-aminoquinoline analogues by modifying its side chain with an extension towards the synthesis of quinoline-acridine conjugates and tested their in vitro antiplasmodial activity against the NF 54 strain of P. falciparum. ${ }^{114}$ Among the tested compounds, the compound $166\left(\mathrm{MIC}=0.125 \mu \mathrm{g} \mathrm{ml}^{-1}\right)$ was nearly as active as CQ $\left(\mathrm{MIC}=0.125 \mu \mathrm{g} \mathrm{ml}^{-1}\right)$ while another $\left(167 ; \mathrm{MIC}=0.031 \mu \mathrm{g} \mathrm{ml}^{-1}\right)$ exhibited four-fold more potency than $\mathbf{C Q}$. Three of the selected test compounds (166, 167 and 168; Fig. 19) with good in vitro antiplasmodial potency were chosen for in vivo screening in Swiss mice infected with the CQ-resistant N-67 strain of P. yoelii. Compound 167 showed a curative response to all treated Swiss mice at doses of $50 \mathrm{mg} \mathrm{kg}^{-1}$ and $25 \mathrm{mg} \mathrm{kg}^{-1}$ for 4 days given via intra-peritoneal route. The compound was orally active at the dose of $100 \mathrm{mg} \mathrm{kg}^{-1}$ and has proved the importance of exploring the 4-aminoquinoline class for the discovery of new antimalarial agents.

The hybrid design strategy was further exemplified by Whitlock and co-workers in the synthesis of CQ-astemizole (non-sedating H1 antagonist) conjugates 170 along with their antiplasmodial evaluation against K1 strain of $P$. falciparum (Fig. 20). ${ }^{115}$ Most of the conjugates were three to four folds more potent than CQ with the most potent of the tested compound exhibiting an $\mathrm{IC}_{50}$ of $23 \mathrm{nM}$. Importantly, the compounds displayed more than 100 times selectivity for antiparasitic activity over cell-based cytotoxicity. Based on the preliminary in vitro $P$. falciparum activity, two potent compounds were further screened for in vivo studies on a $P$. berghei mouse malaria model.

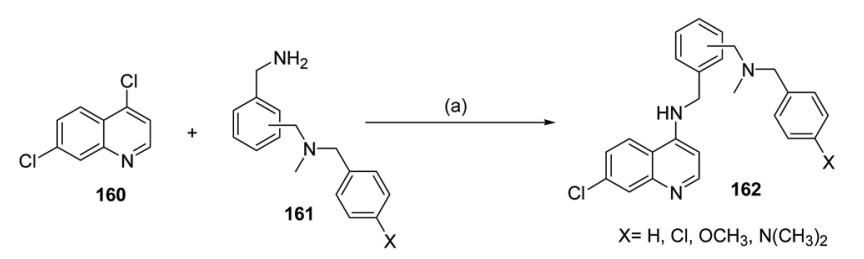

Scheme 26 Reagents and conditions: (a) anhyd. N-methyl-2-pyrrolidine, $\mathrm{K}_{2} \mathrm{CO}_{3}, \mathrm{Et}_{3} \mathrm{~N}$. 


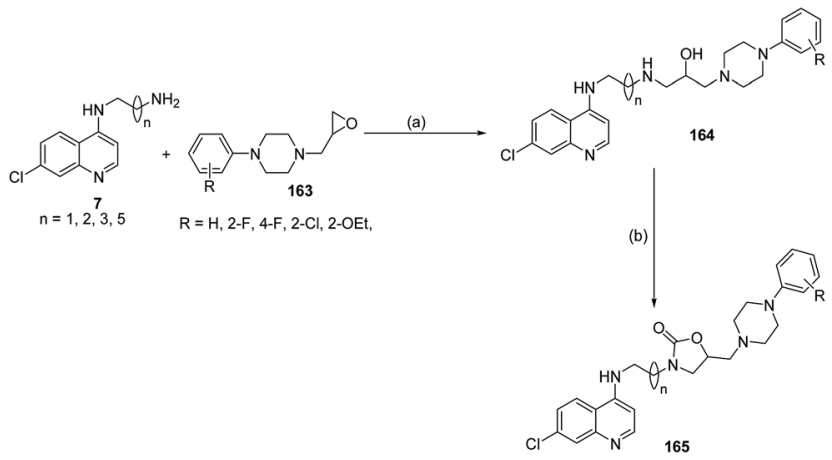

Scheme 27 Reagents and conditions: (a) $\mathrm{MeOH}, 55{ }^{\circ} \mathrm{C}$; (b) trisphosgene, DCM, $0^{\circ} \mathrm{C}-r t$.

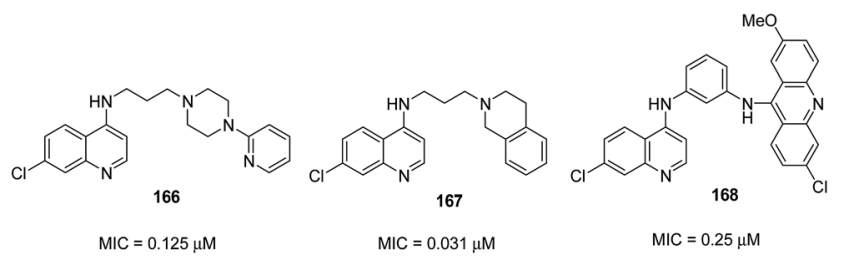

Fig. 19 Most potent compounds among the series.

Santos and colleagues synthesized molecular hybrids of commercially available squarates with the antimalarial drugs, chloroquine (CQ) and primaquine (PM) with improved antiplasmodial activity compared with the squarates. ${ }^{116}$ Three of the synthesized conjugates containing two 7-chloroquinoline moieties were two fold more active than the standard drug, CQ with $\mathrm{IC}_{50}$ values ranging from $0.095-0.20 \mu \mathrm{M}$ against the CQ-resistant W2 strain of $P$. falciparum (Fig. 21). The length of the carbon chain introduced as the linker between the squarates and quinoline scaffolds has been shown to have a major impact on the observed activity profiles with the activity generally increasing with the increase in chain length.

Vacuolar plasmepsins, pepsin-like aspartic proteases present in different species of the parasite Plasmodium, are recognized as promising targets for the enlargement of new antimalarial agents. ${ }^{117}$ They comprise plasmepsins 1 (PLM 1), plasmepsins 2 (PLM 2), plasmepsins 4 (PLM 4) and a histo-aspartic protease

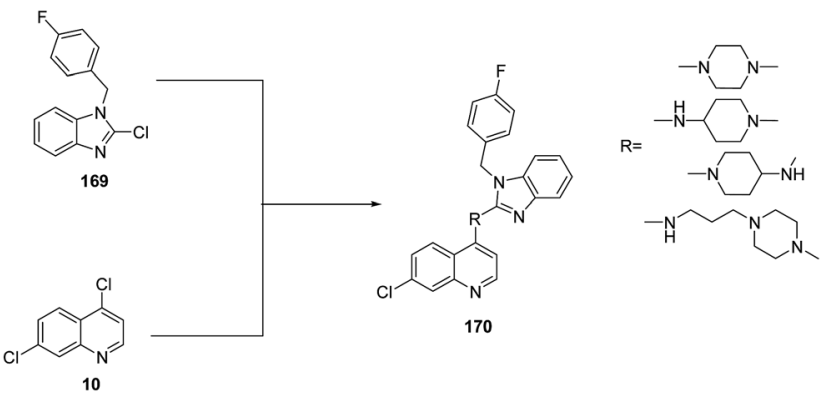

Fig. 20 General strategy of 7-chloroquinoline-astemizole based hybrids.
(HAP) and play a vital role in the survival of the parasite. These enzymes are contained in the food vacuole of the parasite and are directly involved in the degradation of human haemoglobin during the erythrocytic stage. Statin-based molecules are shown to be potent inhibitors of PLMs, however with restricted effectiveness in killing the parasite ( $\mathrm{IC}_{50}$ range $\left.2-20 \mu \mathrm{M}\right){ }^{117}$ In 2012, Romeo and co-workers, in an attempt to circumvent the limitations associated with statin-based molecules, synthesized its hybrids with 4-aminoquinoline $\mathbf{1 7 2 . { } ^ { 1 1 8 }}$ It was considered that since both of these molecules interfere with the haemoglobin catabolism process, the resulting hybrid would have an improvement in overall antimalarial potency. ${ }^{119}$ Two of the 4-aminoquinoline-statin hybrids (172a and 172b) were more potent than CQ against the CQ-resistant strain (Fig. 22) with good selectivity against cathepsin D. It was concluded from $\beta$-hematin inhibitory assay that the 7-chloro group in the quinoline ring is obligatory for the antiplasmodial efficacy of the synthesized conjugates.

Chibale and co-workers reported the preparation of 4-aminoquinoline-3-hydroxypyridin-4-one conjugates on the basis of additive in vitro combination of $\mathrm{N}$-alkyl-3-hydroxypyridin-4-one with chloroquine (CQ) (Scheme 28). All the synthesized conjugates were assessed in vitro against CQ-resistant (K1 and W2) and -sensitive (3D7) strains of $P$. falciparum along with $\beta$-hematin formation studies. ${ }^{120}$ Most of the conjugates were shown to have superior $\beta$-hematin inhibition activity to that of CQ and a strong correlation was observed between antimalarial profiles and inhibition of $\beta$-hematin formation. Among the synthesized conjugates, three potent compounds with longer alkyl chains ( $n=3,5)$ against $\mathrm{K} 1,3 \mathrm{D} 7$, and $\mathrm{W} 2$, respectively, having $\mathrm{IC}_{50} \mathrm{~S}$ of $175 \mathrm{c}(0.13,0.004$, and $0.1 \mu \mathrm{M})$; 175d $(0.08,0.01$, and $0.02 \mu \mathrm{M})$; and $174 \mathrm{~g}(0.07,0.03$, and $0.08 \mu \mathrm{M})$. A cytotoxicity study on a mammalian cell line showed that most of the conjugates screened were non-cytotoxic and far less toxic than podophyllotoxin (POD).

Another attempt to explore the antimalarial potential of bisquinolines was made by N'Da and co-workers by exploring the synthesis of a series of bis-quinolines and bis-pyrrolo[1,2a] quinoxalines containing various polyamine linkers along with the determination of their aqueous solubility and distribution coefficients (Scheme 29). ${ }^{\mathbf{1 2 1}}$ The antimalarial potency of these compounds was assessed against CQ-sensitive (D10) and CQresistant (Dd2) strains of $P$. falciparum besides the growth inhibitory studies against a panel of cancer cell lines. Bisquinolines showed a general increase in antimalarial activity
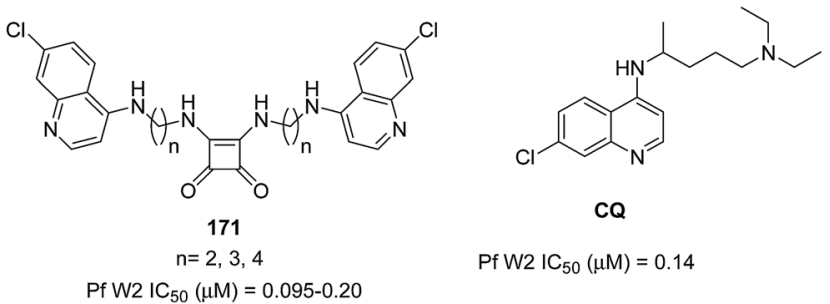

Pf W2 IC $50(\mu M)=0.14$

Fig. 21 Most potent 4-aminoquinoline-squarate hybrids and CQ. 


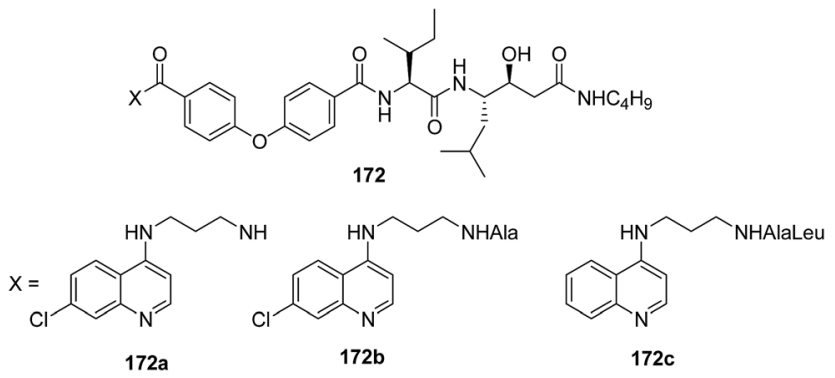

Fig. 22 Most potent 4-aminoquinoline-statin hybrids 171a, 171b, 171c.

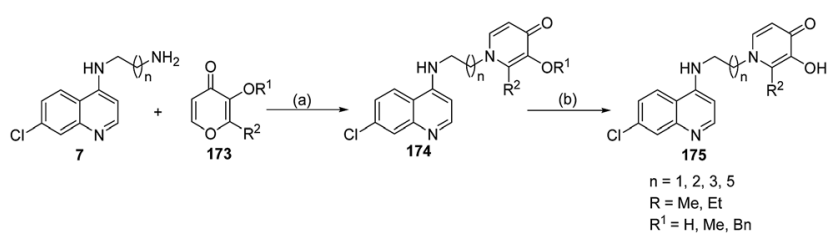

Scheme 28 Reagents and conditions: (a) 50\% aq. EtOH, pH 13 (2 M $\mathrm{NaOH}$ ), $90-120^{\circ} \mathrm{C}, 18-24 \mathrm{~h}$; (b) $\mathrm{H}_{2}, \mathrm{Pd} / \mathrm{C}, 2 \mathrm{M}$ ethanolic $\mathrm{HCl}, 4$ atm or $1: 2: 3 \mathrm{H}_{2} \mathrm{O} / \mathrm{EtOH} / \mathrm{HCl}, 74^{\circ} \mathrm{C}, 24 \mathrm{~h}$.

with the increase in protonation sites given by polyamine linkers that could help to overcome CQ-resistance. The 7-chloro-substituted hybrids have been shown to possess more potency compared to either 5-methyl quinoline or pyrrolo[1,2a] quinoxaline conjugates. Among the bis-quinoline, triethylenetetramine and $N, N$-bis(3-aminopropyl)ethylene-diamine linkers were found to be the most potent of all the synthesized conjugates.

Chibale et al. explored the molecular hybridization paradigm for the synthesis of three groups of hybrid molecules by covalent fusion of azidothymidine (AZT) with dihydroartemisinin (DHA), a tetraoxane or a 4-aminoquinoline derivative that target $P$. falciparum and HIV simultaneously. All synthesized compounds were tested for their inhibitory activity against pseudotyped HIV-1, CQ-sensitive (3D7) and CQ-resistant (Dd2)

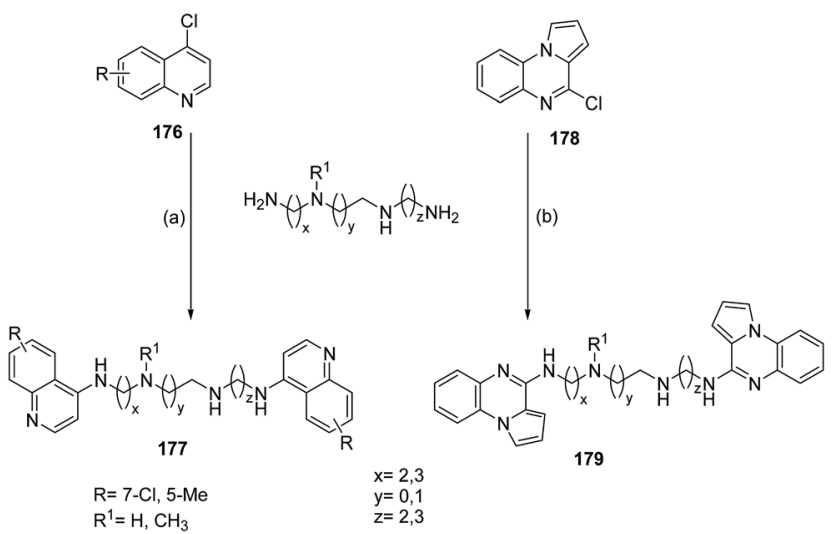

Scheme 29 Reagents and conditions: (a) $135-145{ }^{\circ} \mathrm{C}, 16-20 \mathrm{~h}$; (b) $\mathrm{K}_{2} \mathrm{CO}_{3}, \mathrm{DMF}, 135-145^{\circ} \mathrm{C}, 16 \mathrm{~h}$.

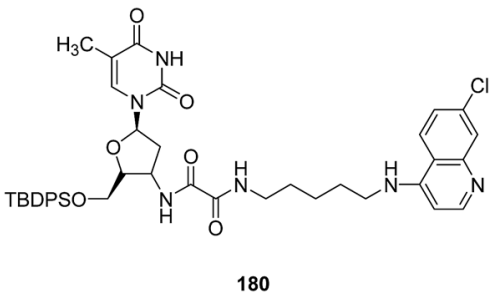

Fig. 23 Most potent CQ-AZT compound 180 .

strains of $P$. falciparum at three different concentrations (50, 5 and $1 \mu \mathrm{M}$ ) along with cytotoxicity assessment against HeLa cells. Among the tested conjugates, a potent CQ-AZT conjugate (180, Fig. 23) exhibited $\mathrm{IC}_{50}$ values of 0.38 and $0.08 \mu \mathrm{M}$ (more potent than CQ and tetraoxane) against 3D7 and Dd2 strains of $P$. falciparum, respectively. This compound also exhibited enhanced inhibitory activity ( $\mathrm{IC}_{50}$ value of $0.9 \mu \mathrm{M}$ ) against pseudotyped HIV-1 with a selective index of 31.8. However, higher cytotoxicity in HeLa cells discontinued its further testing. ${ }^{122}$

In a recent communication, Rao et al. reported the synthesis of a series of 4-aminoquinoline- $4 \mathrm{H}$-chromene conjugates along with their antimalarial evaluation against two $P$. falciparum strains, namely 3D7 (CQ-sensitive) and K1 (CQ-resistant). ${ }^{123}$ All synthesized conjugates possessed activity in the $\mu \mathrm{M}$ range with compounds 181 having piperazine linkage and nitro substituent and $\mathbf{1 8 2}$ with azapane linkage and chloro substituent at the C-6 position of $4 H$-chromene showing comparable activity to that of CQ (Fig. 24). Further, molecular docking suggested that these conjugates exhibited strong binding affinity with $P$. falciparum lactate dehydrogenase (PfLDH) and act as potent inhibitors of the parasite specific glycolytic pathway.

Chibale et al. recently disclosed the synthesis of 4-aminoquinoline-benzoxazole conjugates and screened for their antimalarial efficacy against K1 (multidrug resistant) and NF54 (sensitive) strains of the parasite $P$. falciparum. ${ }^{\mathbf{1 2 4}}$ The antiplasmodial activities of the synthesized compounds exhibited a good structure-activity relationship, which led to the recognition of highly promising conjugates having $\mathrm{IC}_{50} \mathrm{~S}$ in the $\mathrm{nM}$ range against both $\mathrm{K} 1$ and NF54 strains of $P$. falciparum. Further, the synthesized conjugates showed good in vitro microsomal metabolic stability along with desirable in vivo
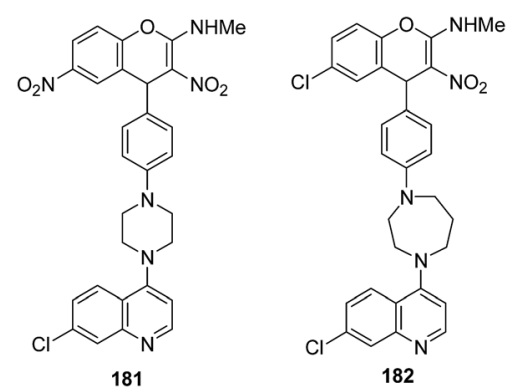

Fig. 24 Most potent 7 -chloroquinoline-4H-chromene conjugates 181 and 182. 

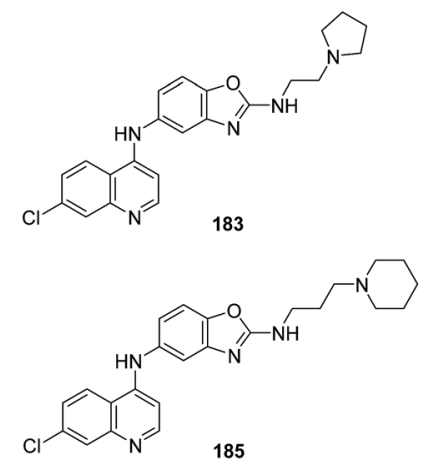
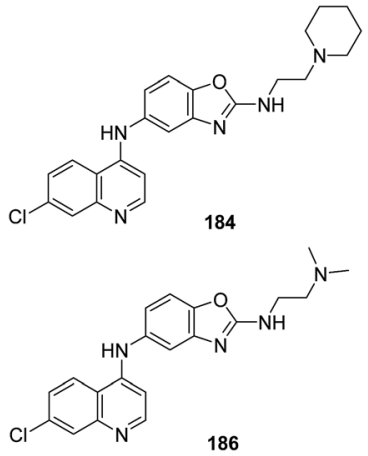

Fig. 25 Most potent 7-chloroquinoline-benzoxazole conjugates 183 , 184,185 and 186.

pharmacokinetic results. The four most promising conjugates (183, 184, 185 and 186, Fig. 25) were subjected to in vivo antimalarial studies against $P$. berghei infected mice. Compound 183 showed good in vivo oral efficacy and completely cured the treated mice at a low multiple dose of $4-10 \mathrm{mg} \mathrm{kg}{ }^{-1}$. Mechanistic studies revealed the inhibition of hemozoin formation as one of the probable mechanisms of action for these compounds. Further, $\beta$-haematin inhibition studies confirmed the importance of the quinoline nucleus for antiplasmodial activity as non-benzoxazole intermediates did not show any inhibitory activity even at highest tested concentration.

Lopez and co-workers in a recent communication showed the synthesis of $3^{\prime}$-dehydroxy-isotebuquine analogs of 4-aminoquinoline 192 via a five step synthetic sequence in good to excellent yields as shown in Scheme 30. The synthesized conjugates were assessed for their $\beta$-hematin inhibitory activity. Among eight of the synthesized conjugates, six compounds were shown to have greater than $97 \%$ IHF value (\% inhibition of hemozoin formation). These potent compounds were further tested for their in vivo antimalarials activity in mice infected with $P$. berghei ANKA CQ-susceptible strain with three of the conjugates displaying in vivo antimalarial efficacy comparable to that of $\mathbf{C Q} .^{125}$

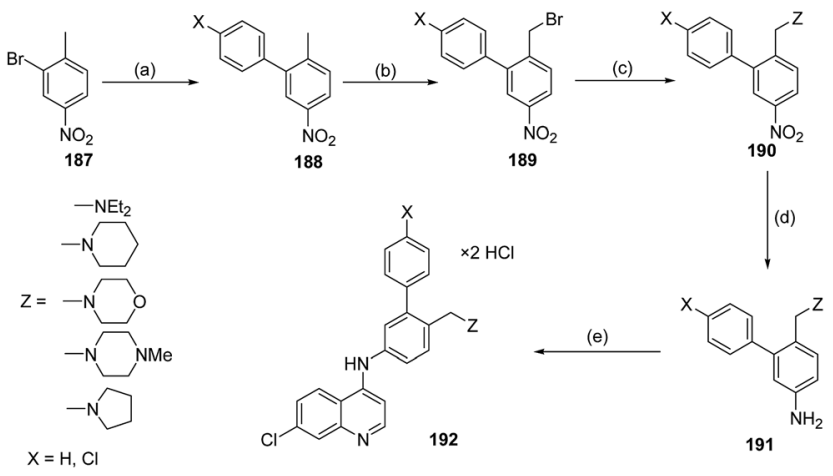

Scheme 30 Reagents and conditions: (a) phenylboronic acid, toluene, $\mathrm{Pd}\left(\mathrm{PPh}_{3}\right)_{4}(5 \%), \mathrm{K}_{2} \mathrm{CO}_{3}, \mathrm{EtOH}: \mathrm{H}_{2} \mathrm{O}(1: 1), 100{ }^{\circ} \mathrm{C}, 24 \mathrm{~h}$; (b) NBS, $\mathrm{CCl}_{4}$, $(\mathrm{PhCO})_{2} \mathrm{O}_{2}$, light, reflux, $24 \mathrm{~h}$; (c) dialkylamine, toluene, reflux, $6 \mathrm{~h}$; (d) $\mathrm{Sn} / \mathrm{HCl}, 70^{\circ} \mathrm{C}, 2 \mathrm{~h}$; (e) 4,7-dichloroquinoline, $\mathrm{EtOH}, \mathrm{HCl}$ (cat.), reflux, $6 \mathrm{~h}$.

\section{Conclusions}

The development of new drugs with improved physicochemical profiles, lack of toxicity, synthetic selectivity and economic accessibility represents a big challenge for the pharmaceutical sector and warrants continuous efforts. The present review establishes the attention of organic medicinal chemists in the field of 4-aminoquinoline-hybridization towards the synthesis of new molecular frameworks for averting and delaying the emergence of drug resistance along with the improvement of efficacy. The validation of molecular hybridization, however, is crucial and the benefit of the hybrid over the separate pharmacophores or their $1: 1$ combination should be confirmed.

\section{Notes and references}

1 T. Bishop and P. Sham, Analysis of Multifactorial Diseases, Academic Press, New York, 2000, pp. 1-320.

2 M. H. Cohen, G. A. Williams, R. Sridhara, G. Chen, W. D. J. McGuinn, D. Morse, S. Abraham, A. Rahman, C. Liang, R. Lostritto, A. Baird and R. Pazdur, Clin. Cancer Res., 2004, 35, 1212.

3 S. Frantz, Nature, 2005, 437, 942.

4 H. Takano, K. Sawada, N. Sato, A. Natoya, T. Tarumi, S. Hirayama, K. Koizumi, T. A. Takahashi, S. Sekiguchi and T. Koike, Leuk. Lymphoma, 1996, 21, 473.

5 B. A. Larder, S. D. Kemp and P. R. Harrigan, Science, 1995, 269, 696.

6 S. A. Eisen, D. K. Miller, R. S. Woodward, E. Spitznagel and T. R. Przybeck, Arch. Intern. Med., 1990, 150, 1881.

7 N. S. Skolnik, J. D. Beck and M. Clark, Am. Fam. Physician, 2000, 61, 3049.

8 G. Glass, Nat. Rev. Drug Discovery, 2004, 3, 731.

9 W. H. Frishman and A. L. Zuckerman, Expert Rev. Cardiovasc. Ther., 2004, 2, 675.

10 N. A. Flores, Curr. Opin. Invest. Drugs, 2004, 5, 984.

11 T. N. C. Wells, P. L. Alonso and W. E. Gutteridge, Nat. Rev. Drug Discovery, 2009, 8, 879.

12 P. G. Clay, T. A. H. Taylor, A. G. Glaros, M. P. McRae, C. Williams, D. McCandless and M. Oelklaus, Ther. Clin. Risk Manage., 2008, 4, 291.

13 R. Morphy and Z. Rankovic, J. Med. Chem., 2005, 48, 6523. 14 B. Meunier, Acc. Chem. Res., 2008, 4, 69.

15 F. W. Muregi and A. Ishih, Drug Dev. Res., 2010, 71, 20.

16 C. Viegas-Junior, A. Danuello, V. D. S. Bolzani, E. J. Barreiro and C. A. M. Fraga, Curr. Med. Chem., 2007, 14, 1829.

17 C. A. M. Fraga, Expert Opin. Drug Discovery, 2009, 4, 605.

18 R. Morphy and Z. Rankovic, Curr. Pharm. Des., 2009, 15, 587.

19 J. O. Gyapong, M. Gyapong, N. Yellu, K. Anakwah, G. Amofah, M. Bockarie and S. Adjiei, Lancet, 2010, 375, 160.

20 World Health Organization, Global report for research on infectious diseases of poverty, 2012, http://whqlibdoc.who.int/ publications/2012/9789241564489_eng.pdf. 
21 WHO, World Malaria Report, 2013, http://www.who.int/ malaria/publications/world malaria report 2013/en/ index.html.

22 R. Banerjee, J. Liu, W. Beatty, L. Pelosof, M. Klemba and D. E. Goldberg, Proc. Natl. Acad. Sci. U. S. A., 2002, 99, 990.

23 N. D. Gamboa de Domínguez and P. J. Rosenthal, Blood, 1996, 87, 4448.

24 P. Loria, S. Miller, M. Foley and L. Tilley, Biochem. J., 1999, 339, 363.

25 C. D. Fitch, R. Chevli, P. Kanjananggulpan, P. Dutta, K. Chevli and A. C. Chou, Blood, 1983, 62, 1165.

26 T. Yoshida and T. C. Migita, J. Inorg. Biochem., 2000, 82, 33. 27 K. A. de Villiers and T. J. Egan, Molecules, 2009, 14, 2868.

28 T. J. Egan and H. M. Marques, Coord. Chem. Rev., 1999, 190, 493.

29 T. J. Egan, R. Hunter, C. H. Kaschula, H. M. Marques, A. Misplon and J. Walden, J. Med. Chem., 2000, 43, 283.

30 M. Schlitzer, Curr. Med. Chem., 2007, 2, 944.

31 V. Narasimhan and A. Attaran, Malar. J., 2003, 2, 8.

32 T. E. Wellems, Science, 2002, 298, 124.

33 A. B. S. Sidhu, D. Verdier-Pinard and D. A. Fidock, Science, 2002, 298, 210.

34 G. Edwards and G. A. Biagini, Br. J. Clin. Pharmacol., 2006, 61, 690.

35 T. K. Mutabingwa, Acta Trop., 2005, 5, 305.

36 R. T. Eastman and D. A. Fidock, Nat. Rev. Microbiol., 2009, 7, 864.

37 A. M. Dondorp, F. Nosten, P. Yi, D. Das, A. P. Phyo, J. Tarning, K. M. Lwin, F. Ariey, W. Hanpithakpong, S. J. Lee, P. Ringwald, K. Silamut, M. Imwong, K. Chotivanich, P. Lim, T. Herdman, S. S. An, S. Yeung, P. Singhasivanon, N. P. Day, N. Lindegardh, D. Socheat and N. J. White, N. Engl. J. Med., 2009, 361, 455.

38 O. Dechy-Cabaret, F. Benoit-Vical, C. Loup, A. Robert, H. Gornitzka, A. Bonhoure, H. Vial, J. F. Magnaval, J. P. Seguela and B. Meunier, Chem.-Eur. J., 2004, 10, 1625.

39 F. Bellot, F. Cosledan, L. Vendier, J. Brocard, B. Meunier and A. Robert, J. Med. Chem., 2010, 53, 4103.

40 A. Kumar, K. Srivastava, S. R. Kumar, M. I. Siddiqi, S. K. Puri, J. K. Saxena and P. M. S. Chauhan, Eur. J. Med. Chem., 2011, 46, 676.

41 S. Manohar, M. Tripathi and D. S. Rawat, Curr. Top. Med. Chem., 2014, 14, 1706.

42 E. M. Guantai, K. Ncokazi, T. J. Egan, J. Gut, P. J. Rosenthal, R. Bhampidipati, A. Kopinathan, P. J. Smith and K. Chibale, J. Med. Chem., 2011, 54, 3637.

43 F. Bellot, F. Cosledan, L. Vendier, J. Brocard, B. Meunier and A. Robert, J. Med. Chem., 2010, 53, 4103.

44 A. Robert, O. Dechy-Cabaret, J. Cazelles and B. Meunier, Acc. Chem. Res., 2002, 35, 167; F. Benoit-Vical, J. Lelievre, A. Berry, C. Deymier, O. Dechy-Cabaret, J. Cazelles, C. Loup, A. Robert, J. F. Magnaval and B. Meunier, Antimicrob. Agents Chemother., 2007, 51, 1463.

45 J. J. Walsh, D. Coughlan, N. Heneghan, C. Gaynor and A. Bell, Bioorg. Med. Chem. Lett., 2007, 17, 3599.
46 I. Opsenica, D. Opsenica, C. A. Lanteri, L. Anova, W. K. Milhous, K. S. Smith and B. A. Salaja, J. Med. Chem., 2008, 51, 6216.

47 S. Gemma, G. Campiani, S. Butini, B. P. Joshi, G. Kukreja, S. S. Coccone, M. Burrutti, M. Persico, V. Nacci, I. Fiorini, E. Novellino, D. Taramerlli, N. Banilico, S. Parapini, V. Yardley, S. Croft, S. K. Maerk, M. Rottman, R. Brun, M. Coletta, S. Marini, G. Guiso, S. Caccia and C. Fattorusso, J. Med. Chem., 2009, 52, 502.

48 I. Chiyanzu, C. Clarkson, P. J. Smith, J. Gut, P. J. Rosenthal and K. Chibale, Bioorg. Med. Chem., 2005, 13, 3249.

49 C. E. Gutteridge, D. A. Nichols, S. M. Curtis, D. S. Thota, J. V. Vo, L. Gerena, G. Montip, C. O. Asher, D. S. Diaz, C. A. DiTusa, K. Smith and A. K. Bhattacharjee, Bioorg. Med. Chem. Lett., 2006, 16, 5682.

50 M. Liu, P. Wilairat and M. L. Go, J. Med. Chem., 2001, 44, 4443.

51 M. Chen, T. G. Theander, S. B. Christensen, L. Hviid, L. Zhai and A. Kharazmi, Antimicrob. Agents Chemother., 1994, 38, 1470.

52 M. Chen, S. B. Christensen, L. Zhai, M. H. Rasmussen, T. G. Theander, S. Frøkjaer, B. Steffansen, J. Davidsen and A. Kharazmi, J. Infect. Dis., 1997, 176, 1327.

53 N. Sriwilaijaroen, M. Liu, M. L. Go and P. Wilairat, Southeast Asian J. Trop. Med. Public Health, 2006, 37, 607.

54 J. N. Dominguez, J. E. Charris, G. Lobo, N. G. de Dominguez, M. M. Moreno, F. Riggione, E. Sanchez, J. Olson and P. J. Rosenthal, Eur. J. Med. Chem., 2001, 36, 555.

55 M. L. Go, M. Liu, P. Wilairat, P. J. Rosenthal, K. J. Saliba and K. Kirk, Antimicrob. Agents Chemother., 2004, 48, 3241.

56 E. M. Guantai, K. Ncokazi, T. J. Egan, J. Gut, P. J. Rosenthal, P. J. Smith and K. Chibale, Bioorg. Med. Chem., 2010, 18, 8243.

57 K. V. Sashidhara, M. Kumar, R. K. Modukuri, R. K. Srivastava, A. Soni, K. Srivastava, S. V. Singh, J. K. Saxena, H. M. Gauniyal and S. K. Puri, Bioorg. Med. Chem., 2012, 20, 2971.

58 K. V. Sashidhara, S. R. Avula, G. R. Palnati, S. V. Singh, K. Srivastava, S. K. Puri and J. K. Saxena, Bioorg. Med. Chem. Lett., 2012, 22, 5455.

59 B. Insuasty, A. Montoya, D. Becerra, J. Quiroga, R. Abonia, S. Robledo, I. D. Vélez, Y. Upegui, M. Nogueras and J. Cobo, Eur. J. Med. Chem., 2013, 67, 252.

60 F. J. Smit and D. D. N'Da, Bioorg. Med. Chem., 2014, 22, 1128.

61 S. Manohar, U. C. Rajesh, S. I. Khan, B. L. Tekwani and D. S. Rawat, ACS Med. Chem. Lett., 2012, 3, 555.

62 K. Singh, H. Kaur, K. Chibale, J. Balzarini, S. Little and P. V. Bharatam, Eur. J. Med. Chem., 2012, 52, 82.

63 S. I. Pretorius, W. J. Breytenbach, C. de Kock, P. J. Smith and D. D. N'Da, Bioorg. Med. Chem., 2012, 21, 269.

64 A. Thakur, S. I. Khan and D. S. Rawat, RSC Adv., 2014, 4, 20729.

65 D. Kumar, S. I. Khan, B. L. Tekwani, P. Ponnan and D. S. Rawat, Eur. J. Med. Chem., 2015, 89, 490. 
66 S. Manohar, S. I. Khan and D. S. Rawat, Chem. Biol. Drug Des., 2011, 78, 124.

67 A. Kumar, K. Srivastava, S. R. Kumar, M. I. Siddiqi, S. K. Puri, J. K. Sexana and P. M. S. Chauhan, Eur. J. Med. Chem., 2011, 46, 676.

68 H. R. Bhat, U. P. Singh, P. Gahtori, S. K. Ghosh, K. Gogoi, A. Prakash and R. K. Singh, New J. Chem., 2013, 37, 2654.

69 H. R. Bhat, U. P. Singh, P. Gahtori, S. K. Ghosh, K. Gogoi, A. Prakash and R. K. Singh, RSC Adv., 2013, 3, 2942.

70 F. A. Rojas Ruiz, R. N. García-Sánchez, S. V. Estupiñan, A. Gómez-Barrio, D. F. Torres Amado, B. M. PérezSolórzano, J. J. Nogal-Ruiz, A. R. Martínez-Fernández and V. V. Kouznetsov, Bioorg. Med. Chem., 2011, 19, 4562.

71 K. Chauhan, M. Sharma, J. Saxena, S. V. Singh, P. Trivedi, K. Srivastava, S. K. Puri, J. K. Saxena, V. Chaturvedi and P. M. S. Chauhan, Eur. J. Med. Chem., 2013, 62, 693.

72 V. R. Solomon, W. Haq, K. Srivastava, S. K. Puri and S. B. Katti, J. Med. Chem., 2007, 50, 394.

73 S. Gemma, G. Campiani, S. Butini, G. Kukreja, S. S. Coccone, B. P. Joshi, M. Persico, V. Nacci, I. Fiorini, E. Novellino, E. Fattorusso, O. Taglialatela-Scafati, L. Savini, D. Taramelli, N. Basilico, S. Parapini, G. Morace, V. Yardley, S. Croft, M. Coletta, S. Marini and C. Fattorusso, J. Med. Chem., 2008, 51, 1278.

74 S. Gemma, C. Camodeca, S. S. Coccone, B. P. Joshi, M. Bernetti, V. Moretti, S. Brogi, M. C. B. de Macros, L. Savini, D. Taramelli, N. Basilico, S. Parapini, M. Rottmann, R. Brun, S. Lamponi, S. Caccia, G. Guiso, R. L. Summers, R. E. Martin, S. Saponara, B. Gorelli, E. Novellino, G. Campiani and S. Butini, J. Med. Chem., 2012, 55, 6948.

75 C. Ohrt, G. D. Willingmyre, P. Lee, C. Knirsch and W. Milhous, Antimicrob. Agents Chemother., 2002, 46, 2518.

76 A. E. Yeo and K. H. Rieckmann, Int. J. Parasitol., 1995, 25, 531.

77 A. B. Sidhu, Q. Sun, L. J. Nkrumah, M. W. Dunne, J. C. Sacchettini and D. A. Fidock, J. Biol. Chem., 2007, 282, 2494.

78 M. Perić, A. Fajdetić, R. Rupčić, S. Alihodžić, D. Ziher, M. Bukvić Krajačić, K. S. Smith, Z. Ivezić-Schönfeld, J. Padovan, G. Landek, D. Jelić, A. Hutinec, M. Mesić, A. Ager, W. Y. Ellis, W. K. Milhous, C. Ohrt and R. Spaventi, J. Med. Chem., 2012, 55, 1389.

79 D. Pesic, K. Starcevic, A. Toplak, E. Herreros, J. Vidal, M. J. Almela, D. Jelic, S. Alihodzic, R. Spaventi and M. Peric, J. Med. Chem., 2012, 55, 3216.

80 P. Singh, P. Singh, M. Kumar, J. Gut, P. J. Rosenthal, K. Kumar, V. Kumar, M. P. Mahajan and K. Bisetty, Bioorg. Med. Chem. Lett., 2012, 22, 57.

81 R. Raj, C. Biot, S. Carrère-Kremer, L. Kremer, Y. Guérardel, J. Gut, P. J. Rosenthal and V. Kumar, Chem. Biol. Drug Des., 2014, 83, 191.

82 P. Singh, R. Raj, P. Singh, J. Gut, P. J. Rosenthal and V. Kumar, Eur. J. Med. Chem., 2014, 71, 128.

83 R. Raj, V. Mehra, J. Gut, P. J. Rosenthal, K. J. Wicht, T. J. Egan, M. Hopper, L. A. Wrischnik, K. M. Land and V. Kumar, Eur. J. Med. Chem., 2014, 84, 425.
84 S. P. Kumar, J. Gut, R. C. Guedes, P. J. Rosenthal, M. M. M. Santos and R. Moreira, Eur. J. Med. Chem., 2011, 46, 927.

85 R. Raj, P. Singh, P. Singh, J. Gut, P. J. Rosenthal and V. Kumar, Eur. J. Med. Chem., 2013, 62, 590.

86 R. Raj, J. Gut, P. J. Rosenthal and V. Kumar, Bioorg. Med. Chem. Lett., 2014, 24, 756.

87 R. Raj, C. Biot, S. Carrère-Kremer, L. Kremer, Y. Guérardel, J. Gut, P. J. Rosenthal, D. Forge and V. Kumar, Chem. Biol. Drug Des., 2014, 83, 622.

88 Nisha, J. Gut, P. J. Rosenthal and V. Kumar, Eur. J. Med. Chem., 2014, 84, 566.

89 G. Gasser and N. Metzler-Nolte, Curr. Opin. Chem. Biol., 2012, 16, 84 .

90 C. Biot, W. Castro, C. Y. Botte and M. Navarro, Dalton Trans., 2012, 41, 6335; C. G. Hartinger and P. J. Dyson, Chem. Soc. Rev., 2009, 38, 391; G. Jaouen, Bioorganometallics: Biomolecules, Labeling, Medicine, WileyVCH Verlag GmbH \& Co. KGaA, Weinheim, 2006.

91 M. A. Jakupec, M. Galanski, V. B. Arion, C. G. Hartinger and B. K. Keppler, Dalton Trans., 2008, 183.

92 J. A. Ocheskey, S. E. Harpstrite, A. Oksman, D. E. Goldberg and V. Sharma, Chem. Commun., 2005, 12, 1622.

93 C. Biot, G. Glorian, L. A. Maciejewski, J. S. Brocard, O. Domarle, G. Blampain, P. Millet, A. J. Georges, H. Abessolo, D. Dive and J. Lebibi, J. Med. Chem., 1997, 40, 3715 .

94 N. Chavain, E. Davioud-Charvet, X. Trivelli, L. Mbeki, M. Rottmann, R. Brun and C. Biot, Bioorg. Med. Chem., 2009, 17, 8048.

95 R. Arancibia, F. Dubar, B. Pradines, I. Forfar, D. Dive, A. H. Klahn and C. Biot, Bioorg. Med. Chem., 2010, 18, 8085. 96 P. F. Salas, C. Herrmann, J. F. Cawthray, C. Nimphius, A. Kenkel, J. Chen, C. de Kock, P. J. Smith, B. O. Patrick, M. J. Adam and C. Orvig, J. Med. Chem., 2013, 56, 1596.

97 C. Herrmann, P. F. Salas, J. F. Cawthray, C. de Kock, B. O. Patrick, P. J. Smith, M. J. Adam and C. Orvig, Organometallics, 2012, 31, 5736.

98 F. Bellot, F. Cosledan, L. Vendier, J. Brocard, B. Menunier and A. Robert, J. Med. Chem., 2010, 53, 4103.

99 L. Glans, D. Taylor, C. de Kock, P. J. Smith, M. Haukka, J. R. Moss and E. Nordlander, J. Inorg. Biochem., 2011, 105, 985.

100 S. D. Khanye, J. Gut, P. J. Rosenthal, K. Chibale and G. S. Smith, J. Organomet. Chem., 2011, 696, 3296.

101 L. Glans, W. Hu, C. Jost, C. de Kock, P. J. Smith, M. Haukka, H. Bruhn, U. Schatzschneider and E. Nordlander, Dalton Trans., 2012, 41, 6443.

102 Y. Li, C. de Kock, P. J. Smith, K. Chibale and G. S. Smith, Organometallics, 2014, 33, 4345.

103 K. Kumar, S. Schniper, A. G. Alez-Sarrías, A. A. Holder, N. Sanders, D. Sullivan, W. L. Jarrett, K. Davis, F. Bai, N. P. Seeram and V. Kumar, Eur. J. Med. Chem., 2014, 86, 81.

104 D. De, F. M. Krogstad, L. D. Byers and D. J. Krogstad, J. Med. Chem., 1998, 41, 4918. 
105 T. S. Feng, E. M. Guantai, M. J. Nell, C. E. J. van Rensburg, H. C. Hoppe and K. Chibale, Bioorg. Med. Chem. Lett., 2011, 21, 2882.

106 M. C. Lombard, D. D. N'Da, J. C. Breytenbach, P. J. Smith and C. A. Lategan, Bioorg. Med. Chem. Lett., 2010, 20, 6975.

107 M. C. Lombard, D. D. N'Da, J. C. Breytenbach, P. J. Smith and C. A. Lategan, Bioorg. Med. Chem. Lett., 2011, 21, 1683. 108 T. S. Feng, E. M. Guantai, M. J. Nell, E. J. Constance, V. Rensburg, K. Ncokazi, T. J. Egan, H. C. Hoppe and K. Chibale, Biochem. Pharmacol., 2011, 82, 236.

109 F. Coslédan, L. Fraisse, A. Pellet, F. Guillou, B. Mordmüller, P. G. Kremsner, A. Moreno, D. Mazier, J. P. Maffrand and B. Meunier, Proc. Natl. Acad. Sci. U. S. A., 2008, 105, 17579.

110 N. C. P. Araújo, V. Barton, M. Jones, P. A. Stocks, S. A. Ward, J. Davies, P. G. Bray, A. E. Shone, M. L. S. Cristiano and P. M. O'Neill, Bioorg. Med. Chem. Lett., 2009, 19, 2038.

111 L. Gupta, K. Srivastava, S. Singh, S. K. Puri and P. M. S. Chauhan, Bioorg. Med. Chem. Lett., 2008, 18, 3306. 112 V. K. Zishiri, M. C. Joshi, R. Hunter, K. Chibale, P. J. Smith, R. L. Summers, R. E. Martin and T. J. Egan, J. Med. Chem., 2011, 54, 6956.

113 F. Kobarfard, V. Yardley, S. Little, F. Daryaee and K. Chibale, Chem. Biol. Drug Des., 2012, 79, 326.

114 A. Kumar, K. Srivastava, S. R. Kumar, S. K. Puri and P. M. S. Chauhan, Bioorg. Med. Chem. Lett., 2010, 20, 7059. 115 C. C. Musonda, G. A. Whitlock, M. J. Witty, R. Brun and M. Kaiser, Bioorg. Med. Chem. Lett., 2009, 19, 481.

116 C. J. A. Ribeiro, S. P. Kumar, J. Gut, L. M. Gonçalves, P. J. Rosenthal, R. Moreira and M. M. M. Santos, Eur. J. Med. Chem., 2013, 69, 365.
117 K. Ersmark, B. Samuelsson and A. Hallberg, Med. Res. Rev., 2006, 26, 626.

118 N. Vaiana, M. Marzahn, S. Parapini, P. Liu, M. Dell'Agli, A. Pancotti, E. Sangiovanni, N. Basilico, E. Bosisio, B. M. Dunn, D. Taramelli and S. Romeo, Bioorg. Med. Chem. Lett., 2012, 22, 5915.

119 M. Mungthin, P. G. Bray, R. G. Ridley and S. A. Ward, Antimicrob. Agents Chemother., 1998, 42, 2973.

120 W. A. Andayi, T. J. Egan, J. Gut, P. J. Rosenthal and K. Chibale, ACS Med. Chem. Lett., 2013, 4, 642.

121 L. van Heerden, J. C. Breytenbach, C. de Kock, P. J. Smith, J. W. Breytenbach, T. T. Cloete and D. D. N'Da, Eur. J. Med. Chem., 2012, 55, 335.

122 M. N. Aminake, A. Mahajan, V. Kumar, R. Hans, L. Wiesner, D. Taylor, C. de Kock, A. Grobler, P. J. Smith, M. Kirschner, A. Rethwilm, G. Pradel and K. Chibale, Bioorg. Med. Chem., 2012, 20, 5277.

123 A. Parthiban, J. Muthukumaran, A. Manhas, K. Srivastava, R. Krishna and H. S. P. Rao, Bioorg. Med. Chem. Lett., 2015, DOI: 10.1016/j.bmcl.2015.08.030.

124 D. S. B. Ongarora, N. Strydom, K. Wicht, M. Njoroge, L. Wiesner, T. J. Egan, S. Wittlin, U. Jurva, C. M. Masimirembwa and K. Chibale, Bioorg. Med. Chem., 2015, 23, 5419.

125 A. H. Romero, M. E. Acosta, N. Gamboa, J. E. Charris, J. Salazar and S. E. Lopez, Bioorg. Med. Chem., 2015, 23, 4755 . 